\title{
Spectral and Energy Spectral Efficiency Optimization 2 of Joint Transmit and Receive Beamforming Based Multi-Relay MIMO-OFDMA Cellular Networks
}

4 Kent Tsz Kan Cheung, Student Member, IEEE, Shaoshi Yang, Member, IEEE, and Lajos Hanzo, Fellow, IEEE

5 Abstract-We first conceive a novel transmission protocol for a 6 multi-relay multiple-input-multiple-output orthogonal frequency7 division multiple-access (MIMO-OFDMA) cellular network based 8 on joint transmit and receive beamforming. We then address 9 the associated network-wide spectral efficiency (SE) and energy 10 spectral efficiency (ESE) optimization problems. More specifically, 11 the network's MIMO channels are mathematically decomposed 12 into several effective multiple-input-single-output (MISO) chan13 nels, which are essentially spatially multiplexed for transmission. 14 Hence, these effective MISO channels are referred to as spatial 15 multiplexing components (SMCs). For the sake of improving the $16 \mathrm{SE} / \mathrm{ESE}$ performance attained, the SMCs are grouped using a 17 pair of proposed grouping algorithms. The first is optimal in the 18 sense that it exhaustively evaluates all the possible combinations 19 of SMCs satisfying both the semi-orthogonality criterion and 20 other relevant system constraints, whereas the second is a lower21 complexity alternative. Corresponding to each of the two grouping 22 algorithms, the pair of SE and ESE maximization problems are 23 formulated, thus the optimal SMC groups and optimal power 24 control variables can be obtained for each subcarrier block. These 25 optimization problems are proven to be concave, and the dual 26 decomposition approach is employed for obtaining their solutions. 27 Relying on these optimization solutions, the impact of various 28 system parameters on both the attainable SE and ESE is char29 acterized. In particular, we demonstrate that under certain condi30 tions the lower-complexity SMC grouping algorithm achieves $\mathbf{9 0 \%}$ 31 of the SE/ESE attained by the exhaustive-search based optimal 32 grouping algorithm, while imposing as little as $3.5 \%$ of the latter 33 scheme's computational complexity.

34 Index Terms-Green communications, spatial multiplexing, 35 beamforming, multi-relay, multiple-input-multiple-output or36 thogonal frequency-division multiple-access (MIMO-OFDMA), 37 fractional programming, dual decomposition, cross-layer design.

Manuscript received November 1, 2013; revised March 16, 2014 and July 29, 2014; accepted August 9, 2014. This research has been funded by the Industrial Companies who are Members of the Mobile VCE, with additional financial support from the UK Government's Engineering \& Physical Sciences Research Council (EPSRC). The financial support of the Research Councils UK (RCUK) under the India-UK Advanced Technology Center (IU-ATC), of the EU under the auspices of the Concerto project, and of the European Research Council's Senior Research Fellow Grant is also gratefully acknowledged. The associate editor coordinating the review of this paper and approving it for publication was L. K. Rasmussen.

The authors are with the School of Electronics and Computer Science, University of Southampton, Southampton SO17 1BJ, U.K. (e-mail: ktkc106@ ecs.soton.ac.uk; shaoshi.yang@ecs.soton.ac.uk; lh@ecs.soton.ac.uk).

Color versions of one or more of the figures in this paper are available online at http://ieeexplore.ieee.org.

Digital Object Identifier 10.1109/TWC.2014.2348996

\section{INTRODUCTION}

$\mathbf{R}$ ECENT wireless mobile broadband standards optionally 39 employ relay nodes (RNs) and multiple-input-multiple- 40 output orthogonal frequency-division multiple-access (MIMO- 41 OFDMA) systems [1], [2] for supporting the ever-growing 42 wireless capacity demands. These systems benefit from a ca- 43 pacity gain increasing roughly linearly both with the num- 44 ber of available OFDMA subcarriers (each having the same 45 bandwidth) as well as with the minimum of the number of 46 transmit antennas (TAs) and receive antennas (RAs). However, 47 this capacity-oriented approach conflicts with the increasing 48 need to reduce the system's carbon footprint [3] as increasing 49 the number of radio frequency (RF) chains and subcarriers will 50 incur additional energy costs. In light of these discussions, the 51 goal of this paper is to formally optimize the spectral efficiency 52 (SE) or energy spectral efficiency (ESE) of the downlink (DL) 53 in a multi-relay MIMO-OFDMA cellular system by intelligently 54 allocating the available power and frequency resources and 55 employing joint transmit and receive beamforming $(B F) . \quad 56$

It is widely acknowledged that under the idealized simplify- 57 ing condition of having perfect channel state information (CSI) 58 at the transmitter, the DL or broadcast channel (BC) capacity 59 [4], [5] may be approached with the aid of dirty paper coding 60 (DPC) [6]. However, the practical implementation of DPC is 61 hampered by its excessive algorithmic complexity upon in- 62 creasing the number of users. On the other hand, BF is an attrac- 63 tive suboptimal strategy for allowing multiple users to share the 64 $\mathrm{BC}$ while resulting in reduced multi-user interference (MUI). 65 A low-complexity transmit-BF technique is the zero-forcing 66 based $\mathrm{BF}$ (ZFBF), which can asymptotically achieve the $\mathrm{BC}$ ca- 67 pacity as the number of users tends to infinity [7]. Furthermore, 68 ZFBF may be readily applied to a system with multiple-antenna 69 receivers through the use of the singular value decomposition 70 (SVD). As a result, the associated MIMO channels may be 71 mathematically decomposed into several effective multiple- 72 input-single-output (MISO) channels, which are termed spatial 73 multiplexing components (SMCs) ${ }^{1}$ in this work. Furthermore, 74 in [8], these SMCs are specifically grouped so that the opti- 75 mal grouping as well as the optimal allocation of the power 76

\footnotetext{
${ }^{1}$ Note that these effective MISO channels are different from the physical MISO channels directly composing the physical MIMO channel. For brevity, we coin the term SMC to emphasize that these effective MISOs will be used for the purpose of spatial multiplexing. A more in-depth discussion regarding the concept of SMCs will be provided in Section III.
} 
77 may be found on each subcarrier block using convex opti78 mization. In contrast to the channel-diagonalization methods 79 of [9], [10], [11], the ZFBF approach does not enforce any 80 specific relationship between the total numbers of TAs and 81 RAs. Therefore, ZFBF is more suitable for practical systems, 82 since the number of TAs at the BS is typically much lower 83 than the total number of RAs of all the active user equipments 84 (UEs). Compared to the random beamforming methods, such 85 as that of [12], ZFBF is capable of completely avoiding the 86 interference, allowing us to formulate our SE/ESE maximiza87 tion (SEM/ESEM) problems as convex optimization problems. 88 Due to its desirable performance versus complexity trade-off, 89 in this paper we employ ZFBF in the context of multi-relay 90 aided MIMO-OFDMA systems, where the direct link between 91 the base station (BS) and the UE may be exploited in conjunc92 tion with the relaying link for further improving the system's 93 performance.

94 We formally define the ESE as a counterpart of the area spec95 tral efficiency (ASE) [13], where the latter has the units of [bits/ $96 \mathrm{sec} / \mathrm{Hz} / \mathrm{m}^{2}$, while the former is measured in $[\mathrm{bits} / \mathrm{sec} / \mathrm{Hz} /$ 97 Joule]. The ESE metric has been justified, for example, in [14]98 [17]. However, these contributions did not consider resource 99 allocation in the context of a MIMO system, and only [17] 100 incorporated relaying. On the other hand, although there are nu101 merous contributions on optimal resource allocation in MIMO 102 systems, they typically only focused on either the SEM (equiv103 alently, the sum-rate maximization) or the power minimization 104 [8], [18]-[21]. For example, the authors of [8] applied BF to 105 a DL cellular system and aimed for minimizing the resultant 106 total transmission power, while simultaneously satisfying the 107 per-user rate requirements. The authors of [19] instead choose 108 to minimize the per-antenna transmission powers, while sat109 isfying both the maximum per-antenna power constraints as 110 well as the per-user signal-to-noise-plus-interference (SINR) 111 requirements. Although there exists some literature studying 112 the ESE of relay-aided MIMO systems [22], [23], these con113 tributions typically focus their attention on a simple three114 node network consisting of the source, the destination and a 115 single RN.

116 To summarize, there is a paucity of literature on the convex 117 optimization approach to the ESEM problem associated with 118 both resource allocation and joint transmit/receive beamform119 ing in the context of multi-user multi-relay MIMO-OFDMA 120 systems. Additionally, the Charnes-Cooper transformation [24] 121 is employed in this paper for solving the associated ESEM 122 problem, in contrast to the scalarization approach [25] that 123 requires the weighting of multiple objectives. On the other 124 hand, the Dinkelbach's method [14], [17], [26], [27] is avoided 125 as it would require solving a series of parametric convex 126 problems, rather than the resultant single convex problem of the 127 Charnes-Cooper transformation. Although the latter approach 128 does impose an additional linear constraint on the problem, in 129 our experience, this only marginally increases the complexity 130 of the solution algorithm. The authors of [28] employed the 131 Charnes-Cooper variable transformation for the ESEM of a 132 simple point-to-point link. However, as far as we are aware, the 133 Charnes-Cooper transformation has rarely been used in other 134 contexts for solving the ESEM problem.
Let us now summarize the above discussions and provide a 135 concise list of the novel contributions of this paper:

- We consider a generalized multi-user multi-relay as- 137 sisted MIMO-OFDMA cellular system model for the 138 SEM/ESEM problems. To provide some justification, 139 this system model accounts for both the direct links be- 140 tween the BS and the UEs, as well as the relaying links 141 employing the decode-and-forward (DF) relaying protocol 142 [29]. This system model is unlike that of [7], [8], which 143 did not consider relaying, and it is also distinct from that 144 of [22], [23], which only consider a single $\mathrm{RN}$ and a 145 single UE. Additionally, we dispense with the constraint 146 that the number of antennas at the BS needs to be greater 147 or equal to the sum of the number of antennas at the 148 UEs, which was assumed in [9]-[11]. Furthermore, this 149 system model is built upon our previous work [17] as the 150 network elements may now be equipped with an arbitrary 151 number of antennas for improving the system's SE or ESE 152 performance. 153

- A sophisticated novel transmission protocol is proposed 154 for improving the system's SE/ESE performance. Since 155 the multi-relay MIMO-OFDMA system model considered 156 has not been studied in the context of the SEM/ESEM 157 problems before, we develop a novel transmission protocol 158 that exploits spatial multiplexing in both transmission 159 phases while allowing both the direct and relaying links to 160 be simultaneously active. Although this protocol does not 161 benefit from a higher spatial degree of freedom than that of 162 the conventional half-duplex relay based cooperative sys- 163 tem, we glean more flexibility in choosing the best group 164 of channels for each transmission phase, which leads to 165 additional selection diversity. As a result, the achievable 166 SE/ESE performance may be improved. Again, this pro- 167 tocol is distinct from that presented in [7], [8], since re- 168 laying is not considered in those works. Another benefit is 169 that since spatial multiplexing is employed in conjunction 170 with OFDMA, multiple data streams may be served using 171 the same subcarrier block, while the transmit ZFBF is 172 employed for avoiding the interference. Furthermore, the 173 receive-BF matrices are designed with the aim of generat- 174 ing a number of SMCs that may be grouped for the purpose 175 of increasing the attainable spatial multiplexing gain. 176

- Two SMC grouping algorithms are proposed. To elabo- 177 rate, we present a pair of novel algorithms for grouping the 178 SMCs transmissions. The possibility of relayed transmis- 179 sions means that we have to partition each transmission 180 period into two halves, one consisting of BS-to-UE and 181 BS-to-RN links, and the other consisting of additional BS- 182 to-UE as well as RN-to-UE links. As a result, the SMC- 183 pairs of the two-hop relaying links are incomparable to the 184 SMCs of the direct links in either the first or the second 185 transmission phases. This is because, firstly the RNs are 186 subject to their individual maximum transmission power 187 constraints, and secondly they employ the DF protocol, 188 which means that the information conveyed on the RN- 189 to-UE link cannot be more than that conveyed on the 190 BS-to-RN link. These challenging issues are resolved 191 
by the proposed grouping algorithms. The first grouping algorithm is optimal in the sense that it is based on exhaustive search over all the SMC groupings that satisfy the semi-orthogonality criterion, while the second algorithm constitutes a lower-complexity alternative. This complexity-reduction is achieved by a multi-stage SMC group construction process. In each stage, we firstly compute the orthogonal components with respect to the vectors contained in the tentative SMC group to be constructed using all the residual legitimate SMC vectors, and then insert the particular SMC vector that results in the orthogonal component having the highest norm into the SMC group to be constructed. In principle, this method is similar to that of [7], [8], but it has been appropriately adapted for the multi-relay cellular network considered under the abovementioned particular constraints.

- The problems of choosing the SE- or ESE-optimal SMC groupings and their associated power control values are formulated and solved using convex optimization. In contrast to [8], [18]-[21], the crucial objective of maximizing the ESE metric is employed, as motivated above. On the other hand, in contrast to [14]-[16], we consider a system that allows for simultaneous direct and relayed transmissions for the sake of increasing the attainable spatial multiplexing gain. Although there exist other methods of solving this ESEM problem [14], [17], [25], [27], we employ the Charnes-Cooper transformation [24] for obtaining the maximum ESE solution, as it exhibits a reduced complexity from having to solve only a single convex optimization problem.

222 The rest of this paper is organized as follows. Section II de223 scribes the multi-relay MIMO-OFDMA cellular network con224 sidered, while Section III characterizes our novel transmission 225 protocol that allows for both direct and relaying links to be 226 simultaneously and continuously activated. In Section IV, we 227 elaborate on the aforementioned SMC grouping algorithms 228 conceived for forming the sets of possible SMC transmission 229 groups. The issue of finding the optimal SMC transmission 230 groups and the optimal power control variables is then for231 mulated as an optimization problem in Section $\mathrm{V}$, which is 232 then solved by using a number of variable transformations 233 and relaxations. The performance of both our SMC grouping 234 algorithms and of the SEM/ESEM solvers are characterized 235 in Section VI. Finally, we present our conclusions and future 236 research ideas in Section VII.

\section{7 \\ II. SySTEM MODEL}

238 We focus our attention on the DL of a multi-relay MIMO239 OFDMA cellular network, as shown in Fig. 1. The BS, $M$ DF240 assisted RNs and $K$ UEs are each equipped with $N_{B}, N_{R}$ and $241 N_{U}$ antennas, respectively. The cellular system has access to $242 N$ subcarrier blocks, each encompassing $W$ Hertz of wireless 243 bandwidth. The subcarrier blocks considered here are similar 244 to the resource blocks in the LTE-nomenclature [30]. The BS 245 is located at the cell-center, while the RNs are each located at a 246 fixed distance from the BS and are evenly spaced around it. The

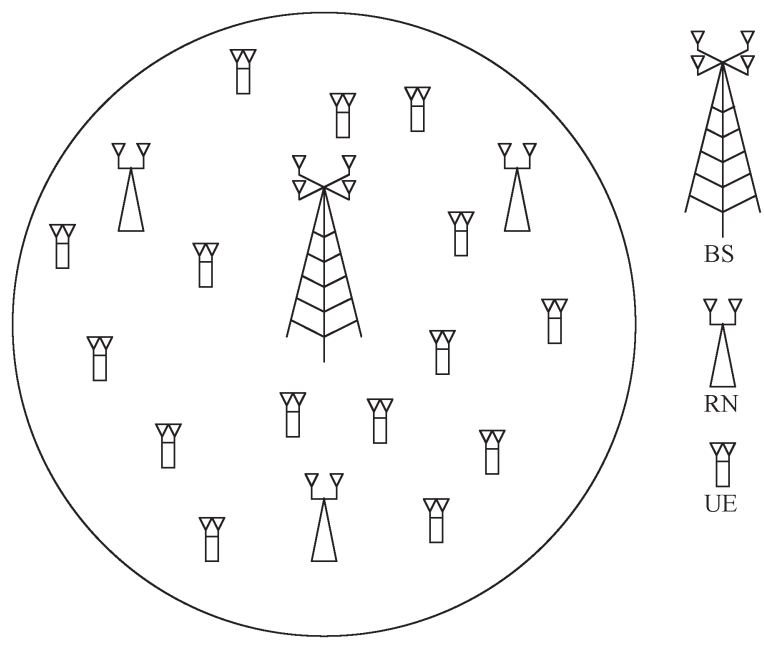

Fig. 1. An example of a multi-relay MIMO-OFDMA cellular network, containing a BS at the cell-center, 3 RNs and 15 UEs.

ratio of the distance between the BS and RNs to the cell radius 247 is denoted by $D_{r}$. On the other hand, the UEs are uniformly 248 distributed in the cell. The BS coordinates and synchronizes its 249 own transmissions with that of the RNs, which employ the DF 250 protocol and thus avoids the problem of noise amplification. As 251 it will be shown in Section V-C-1, this strategy results in a sim- 252 ple algorithm for finding the optimal power control variables. 253

For the subcarrier block $n \in\{1, \ldots, N\}$, let us define the 254 complex-valued wireless channel matrices between the BS and 255 UE $k \in\{1, \ldots, K\}$, between the BS and RN $m \in\{1, \ldots, M\}, 256$ and between RN $m$ and UE $k$ as $\mathbf{H}_{n, k}^{B U} \in \mathbb{C}^{N_{U} \times N_{B}}, \mathbf{H}_{n, m}^{B R} \in 257$ $\mathbb{C}^{N_{R} \times N_{B}}$ and $\mathbf{H}_{n, m, k}^{R U} \in \mathbb{C}^{N_{U} \times N_{R}}$, respectively. These complex- 258 valued channel matrices account for both the frequency-flat 259 Rayleigh fading and the path-loss between the corresponding 260 transceivers. The coherence bandwidth of each wireless link 261 is assumed to be sufficiently high, so that each individual 262 subcarrier block experiences frequency flat fading, although the 263 level of fading may vary from one subcarrier block to another in 264 each transmission period. Additionally, the transceivers are sta- 265 tionary or moving slowly enough so that the level of fading may 266 be assumed to be fixed for the duration of a scheduled trans- 267 mission period. Furthermore, the RAs are spaced sufficiently 268 far apart, so that each TA/RA pair experiences independent and 269 identically distributed (i.i.d.) fading. Since these channels are 270 slowly varying, the system is capable of exploiting the benefits 271 of channel reciprocity associated with time-division duplexing 272 (TDD), so that the CSI becomes available at each BS- and 273 RN-transmitter and at each possible RN- and UE-receiver. To 274 elaborate, $\mathbf{H}_{n, k}^{B U}$ and $\mathbf{H}_{n, m}^{B R}$ are known at the $\mathrm{BS}, \mathbf{H}_{n, m}^{B R}$ and 275 $\mathbf{H}_{n, m, k}^{R U}$ are known at the RN $m$, while $\mathbf{H}_{n, k}^{B U}$ and $\mathbf{H}_{n, m, k}^{R U}$ are 276 also known at UE $k$. Additionally, through the use of dedicated 277 low-rate error-free feedback channels, $\mathbf{H}_{n, m, k}^{R U}$ is also assumed 278 to be known at the BS so that the BS may perform network- 279 wide scheduling. ${ }^{2}$ These channel matrices are assumed to have 280

${ }^{2}$ In this paper, since our focus is on the resource allocation and the associated SE/ESE optimization problems, the idealized simplifying assumption of the availability of perfect CSI is employed. At the current stage, accounting for erroneous CSI using, for example, robust optimization [31] is beyond the scope of this paper and may be addressed in our future work. 
281 full row rank, which may be achieved with a high probability 282 for typical DL wireless channel matrices.

283 Furthermore, each receiver suffers from additive white 284 Gaussian noise (AWGN) having a power spectral density of $N_{0}$. 285 The maximum instantaneous transmission power available to 286 the BS and to each RN due to regulatory and health-constraints 287 is $P_{\max }^{B}$ and $P_{\max }^{R}$, respectively. Since OFDMA modulation 288 constitutes a linear operation, we focus our attention on a single 289 subcarrier block and as usual, we employ the commonly-used 290 equivalent baseband signal model. ${ }^{3}$

\section{III. TRANSMISSION PROTOCOL DESIGN}

292 The system can simultaneously use two transmission modes 293 to convey information to the UEs, namely the BS-to-UE mode, 294 and the relaying-based BS-to-RN and RN-to-UE mode. Note 295 that although in classic OFDMA each data stream is orthogonal 296 in frequency, for the sake of further improving the system's 297 attainable SE or ESE performance, our system employs spatial 298 multiplexing in conjunction with $\mathrm{ZFBF}$ so that multiple data 299 streams may be served using the same subcarrier block, without 300 suffering from interference. Additionally, since the relaying301 based transmission can be split into two phases, the design 302 philosophy of the BF matrices in each phase are described 303 separately, although for simplicity we have assumed that the 304 respective channel matrices remain unchanged in both phases. 305 Firstly, the definition of the semi-orthogonality criterion is 306 given as follows [7].

307 Definition 1: A pair of MISO channels, represented by the 308 complex-valued column vectors $\mathbf{v}_{1}$ and $\mathbf{v}_{2}$, are said to be semi309 orthogonal to each other with parameter $\alpha \in[0,1]$, when

$$
\frac{\left|\Re\left(\mathbf{v}_{1}^{\mathrm{H}} \mathbf{v}_{2}\right)\right|}{\left\|\mathbf{v}_{1}\right\|\left\|\mathbf{v}_{2}\right\|} \leq \alpha .
$$

310 To be more specific, a measure of the grade of orthogonality 311 between $\mathbf{v}_{1}$ and $\mathbf{v}_{2}$ is given by the left-hand side of inequality 312 (1), which ranges from 0 for orthogonal vectors to 1 for linearly 313 dependent vectors.

314 The authors of [7] demonstrated that employing the ZFBF 315 strategy for MISO channels that satisfy $\alpha \rightarrow 0$, while the num316 ber of users obeys $K \rightarrow \infty$, asymptotically achieves the DPC 317 capacity, and it is therefore optimal for the BC channel. Similar 318 principles are followed when maximizing the SE or ESE of the 319 system considered in this paper.

\section{A. BF Design for the First Transmission Phase}

321 In the first transmission phase, only the BS is transmitting, 322 while both the RNs and the UEs act as receivers. This is 323 similar to the classic DL multi-user MIMO model. As described 324 above, our aim is 1) to design a ZFBF matrix for the BS 325 to avoid interference between data streams, and 2) to design

\footnotetext{
${ }^{3}$ Since the specific signal model expressions of each link is dependent on the transmission protocol to be designed, they are not presented here but instead detailed in Section III.
}

receive $\mathrm{BF}$ matrices for the UEs and RNs so that the resultant 326 effective DL channel matrices contain as many semi-orthogonal 327 rows as possible that satisfy (1) for a given $\alpha$. Ideally, all 328 receivers (UEs and RNs) should jointly compute ${ }^{4}$ their receive 329 $\mathrm{BF}$ matrices to accomplish the second goal. However, this 330 is generally impossible, since we cannot realistically assume 331 that the channel matrices associated with each UE and RN 332 are shared among them, due to the geographically-distributed 333 nature of the UEs and RNs. As a compromise, we opt for 334 guaranteeing that each individual effective DL channel matrix 335 contains locally orthogonal rows by employing the SVD [7], 336 [8]. Although these locally orthogonal rows may not remain 337 orthogonal globally, they can be characterized using the semi- 338 orthogonality metric of (1).

Bearing this in mind, the channel matrices of all DL 340 transmissions originating from the BS are decomposed at 341 the BS, UEs and RNs using the SVD [32] as $\mathbf{H}_{n, k}^{B U}=342$ $\mathbf{U}_{n, k}^{B U} \mathbf{S}_{n, k}^{B U}\left(\mathbf{V}_{n, k}^{B U}\right)^{\mathrm{H}}$ and $\mathbf{H}_{n, m}^{B R}=\mathbf{U}_{n, m}^{B R} \mathbf{S}_{n, m}^{B R}\left(\mathbf{V}_{n, m}^{B R}\right)^{\mathrm{H}}$, respec- 343 tively. Thus, the receive-BF matrices for $\mathrm{UE} k$ and $\mathrm{RN} m 344$ are given by $\mathbf{R}_{n, k}^{B U, T_{1}}=\left(\mathbf{U}_{n, k}^{B U}\right)^{\mathrm{H}} \in \mathbb{C}^{N_{U} \times N_{U}}$ and $\mathbf{R}_{n, m}^{B R, T_{1}}=345$ $\left(\mathbf{U}_{n, m}^{B R}\right)^{\mathrm{H}} \in \mathbb{C}^{N_{R} \times N_{R}}$, and the effective DL channel matrices 346 are then given ${ }^{5}$ by $\underline{\mathbf{H}}_{n, k}^{B U, T_{1}}=\mathbf{R}_{n, k}^{B U, T_{1}} \mathbf{H}_{n, k}^{B U}=\mathbf{S}_{n, k}^{B U}\left(\mathbf{V}_{n, k}^{B U}\right)^{\mathrm{H}} \in 347$ $\mathbb{C}^{N_{U} \times N_{B}}$ and $\underline{\mathbf{H}}_{n, m}^{B R, T_{1}}=\mathbf{R}_{n, m}^{B R, T_{1}} \mathbf{H}_{n, m}^{B R}=\mathbf{S}_{n, m}^{B R}\left(\mathbf{V}_{n, m}^{B R}\right)^{\mathrm{H}} \in 348$ $\mathbb{C}^{N_{R} \times N_{B}}$, respectively. Since $\mathbf{V}_{n, k}^{B U}$ and $\mathbf{V}_{n, m}^{B R}$ are both unitary, 349 while $\mathbf{S}_{n, k}^{B U}$ and $\mathbf{S}_{n, m}^{B R}$ are both real and diagonal, these effective 350 DL channel matrices respectively consist of $\min \left(N_{B}, N_{U}\right)$ and 351 $\min \left(N_{B}, N_{R}\right)$ orthogonal non-zero rows ${ }^{6}$ with norms equal 352 to their corresponding singular values. We refer to these non- 353 zero orthogonal rows as the SMCs of their associated MIMO 354 channel matrix. ${ }^{7}$ The $K$ BS-to-UE MIMO channel matri- 355 ces and $M$ BS-to-RN channel matrices generate a total of 356 $\left[K \cdot \min \left(N_{B}, N_{U}\right)+M \cdot \min \left(N_{B}, N_{R}\right)\right]$ SMCs. Since these 357 SMCs are generated from independent MIMO channel matri- 358 ces associated with geographically distributed UEs and RNs, 359 they are not all guaranteed to be orthogonal to each other. 360 Furthermore, since each UE or RN has multiple antennas and 361 $N_{B}$ might not be sufficiently large to simultaneously support 362 all UEs and RNs, we have to determine which specific SMCs 363 should be served. As a result, for each two-phase transmis- 364 sion period, we opt for selecting a SMC group accounting 365 for both phases from the set of available SMC groups. This 366 selection process is achieved by jointly using the SMC group- 367 ing algorithm and solving the optimization problem detailed 368 below. For the sake of clarity, the concepts of the SMC, of 369 the SMC group and of the set of SMC groups are illustrated 370 in Fig. 2.

To elaborate a little further, a set of SMC groups, $\mathcal{G}_{n}, 372$ which is associated with subcarrier block $n$, may be obtained 373

\footnotetext{
${ }^{4}$ The joint computation is required only for attaining the highest number of semi-orthogonal rows globally.

${ }^{5}$ Note that $T_{1}$ is used for indicating the first transmission phase, and underline is used to denote the effective DL channel matrices.

${ }^{6}$ The reason why we use $\min \left(N_{B}, N_{U}\right)$ and $\min \left(N_{B}, N_{R}\right)$, instead of $N_{U}$ and $N_{R}$, is so that the antenna configuration $N_{B} \leq N_{U}$ and/or $N_{B} \leq N_{R}$ is also covered.

${ }^{7}$ Note that only when $N_{B} \geq N_{U}$ and $N_{B} \geq N_{R}$, a single SMC is generated for each receive antenna.
} 


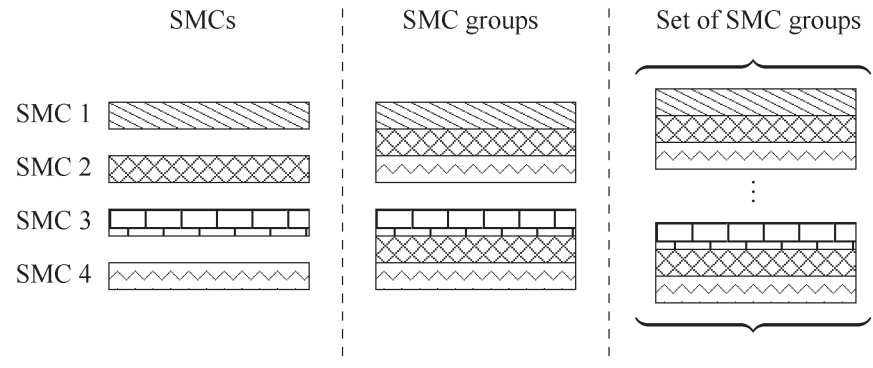

Fig. 2. A conceptual illustration of the differences between SMCs, SMC groups and a set of SMC groups.

374 using one of the grouping algorithms presented in Section IV. 375 The BS selects a single group, $j \in \mathcal{G}_{n}$, containing (but not 376 limited to $)^{8} Q_{j}^{T_{1}}$ SMCs out of the $\left[K \cdot \min \left(N_{B}, N_{U}\right)+M\right.$. $377 \min \left(N_{B}, N_{R}\right)$ ] available $\mathrm{SMCs}$ to be supported by using $378 \mathrm{ZFBF}$. Thus, we have $Q_{j}^{T_{1}} \leq \min \left[N_{B}, K \cdot \min \left(N_{B}, N_{U}\right)+M\right.$. $\left.379 \min \left(N_{B}, N_{R}\right)\right]$ and a multiplexing gain of $Q_{j}^{T_{1}}$ is achieved. 380 Let us denote the refined effective DL channel matrix with 381 rows being the $Q_{j}^{T_{1}}$ selected SMCs as $\underline{\mathbf{H}}_{n, j}^{T_{1}} \in \mathbb{C}^{Q_{j}^{T_{1}} \times N_{B}}$. The $382 \mathrm{ZFBF}$ transmit matrix applied at the BS to subcarrier block $n$ 383 is then given by the following right inverse $\mathbf{T}_{n, j}^{T_{1}}=\left(\underline{\mathbf{H}}_{n, j}^{T_{1}}\right)^{\mathrm{H}}$ $384\left[\underline{\mathbf{H}}_{n, j}^{T_{1}}\left(\underline{\mathbf{H}}_{n, j}^{T_{1}}\right)^{\mathrm{H}_{1}}\right]^{-1} \in \mathbb{C}^{N_{B} \times Q_{j}^{T_{1}}}$. Since $\underline{\mathbf{H}}_{n, j}^{T_{1}} \mathbf{T}_{n, j}^{T_{1}}=\mathbf{I}_{N_{B}}$, the 385 potential interference between the $Q_{j}^{T_{1}}$ selected SMCs is com386 pletely avoided. Furthermore, the columns of $\mathbf{T}_{n, j}^{T_{1}}$ are normal387 ized by multiplying the diagonal matrix $\mathbf{W}_{n, j}^{T_{1}}$ on the right-hand 388 side of $\mathbf{T}_{n, j}^{T_{1}}$ to ensure that each SMC transmission is initially 389 set to unit power. ${ }^{9}$

390 Then, $\mathbf{T}_{n, j}^{T_{1}} \mathbf{W}_{n, j}^{T_{1}}$ is used as the DL transmit-BF matrix 391 for the BS in the first phase. Thus, the effective channel392 to-noise ratios (CNRs) in the first transmission phase can 393 be written as $G_{n, j, e_{1}}^{B U, T_{1}}=\left|w_{n, j, e_{1}}^{B U, T_{1}}\right|^{2} / \Delta \gamma N_{0} W$ and $G_{n, j, e}^{B R, T_{1}}=$ $394\left|w_{n, j, e}^{B R, T_{1}}\right|^{2} / \Delta \gamma N_{0} W$, respectively, where $w_{n, j, e_{1}}^{B U, T_{1}}$ and $w_{n, j, e}^{B R, T_{1}}$ 395 are the diagonal elements in $\mathbf{W}_{n, j}^{T_{1}}$. More specifically, these 396 diagonal elements correspond to SMC group $j$ and sub397 carrier block $n$, and they are associated with either a di398 rect BS-to-UE SMC or a BS-to-RN SMC. The additional 399 subscripts $e_{1} \in\left\{0, \ldots, \min \left[N_{B}, K \cdot \min \left(N_{B}, N_{U}\right)\right]\right\}$ and $e \in$ $400\left\{0, \ldots, \min \left[N_{B}, M \cdot \min \left(N_{B}, N_{R}\right), K \cdot \min \left(N_{R}, N_{U}\right)\right]\right\}$ are 401 used for distinguishing the multiple selected SMCs of the 402 direct links (i.e. those related to UEs), from the multiple se403 lected SMC-pairs ${ }^{10}$ that may be associated with a particular $404 \mathrm{RN} \mathcal{M}(e)$, respectively. Note that $\mathcal{M}(e)$ is a function of $e$, 405 representing the $\mathrm{RN}$ index (similar to $m$ used before) associated 406 with the SMC-pair $e$, as further detailed in Section IV.

\footnotetext{
${ }^{8}$ The SMC group selection, as a part of the scheduling operation, is carried out at the BS before initiating the first transmission phase. Hence, the selected SMC group will also contain $Q_{j}^{T_{2}}$ SMCs selected by the BS for the second transmission phase, as detailed in Section III-B.

${ }^{9}$ Each diagonal element of $\mathbf{W}_{n, j}^{T_{1}}$ is equal to the reciprocal of the norm of the column vector to be normalized.

${ }^{10}$ A single SMC-pair consists of a SMC for the first phase and another for the second phase. Although these SMCs are generated separately in each phase, the SMC-pair associated with a common RN has to be considered as a single entity in the SMC grouping algorithms presented in Section IV.
}

At a given bit-error rate (BER) requirement, $\Delta \gamma$ is the 407 signal-to-noise ratio (SNR) gap between the lower-bound SNR 408 required for achieving the discrete-input-continuous-output 409 memoryless channel (DCMC) capacity and the actual higher 410 SNR required by the modulation/coding schemes of the practi- 411 cal physical layer transceivers employed. For example, making 412 the simplifying assumption that idealized transceivers capable 413 of achieving exactly the DCMC capacity are employed, then 414 $\Delta \gamma=0 \mathrm{~dB}$. Although, strictly speaking, so far it is not possible 415 to operate exactly at the DCMC channel capacity, there does 416 exist several physical layer transceiver designs that operate very 417 close to it [33]. Furthermore, the noise power received on each 418 subcarrier block is given by $N_{0} W$.

\section{B. BF Design in the Second Transmission Phase}

The second transmission phase may be characterized by 421 the MIMO interference channel. A similar methodology is 422 employed in the second transmission phase, except that now 423 both the BS and the RNs are transmitters, while a number of 424 UEs are receiving. In this phase, our aim is 1) to design ZFBF 425 matrices for the BS and RNs to avoid interference between 426 data streams, 2) and to design a receive-BF matrix for each UE 427 so that the effective channel matrices associated with each of 428 its transmitters contain rows which satisfy the semi-orthogonal 429 condition (1) for a given $\alpha$. This means that more data streams 430 may be served simultaneously, thus improving the attainable 431 SE or ESE performance. Since there are multiple distributed 432 transmitters/MIMO channel matrices associated with each UE, 433 the SVD method described in Section III-A, which is performed 434 in a centralized fashion, cannot be readily applied at the 435 transmitter side. Instead, we aim for minimizing the resultant 436 correlation between the generated SMCs, thus increasing the 437 number of SMCs which satisfy (1) for a given $\alpha$. To accomplish 438 this goal, we begin by introducing the shorthand of $\underline{\mathbf{H}}_{n, k}^{B U, T_{2}}=439$ $\mathbf{R}_{n, k}^{U, T_{2}} \mathbf{H}_{n, k}^{B U} \in \mathbb{C}^{N_{U} \times N_{B}}$ and $\underline{\mathbf{H}}_{n, m, k}^{R U, T_{2}}=\mathbf{R}_{n, k}^{U, T_{2}} \mathbf{H}_{n, m, k}^{R U} \in \mathbb{C}^{N_{U} \times N_{R}} 440$ as the effective channel matrices between the BS and UE $k, 441$ and between RN $m$ and UE $k$, respectively, on subcarrier block 442 $n$ in the second transmission phase, where $\mathbf{R}_{n, k}^{U, T_{2}} \in \mathbb{C}^{N_{U} \times N_{U}} 443$ is the yet-to-be-determined UE $k$ 's receive-BF matrix. In light 444 of the preceding discussions, one of our aims is to design 445 $\mathbf{R}_{n, k}^{U, T_{2}}$ so that the off-diagonal values of the matrices given by 446 $\mathbf{A}_{0}=\underline{\mathbf{H}}_{n, k}^{B U, T_{2}}\left(\underline{\mathbf{H}}_{n, k}^{B U, T_{2}}\right)^{\mathrm{H}}=\mathbf{R}_{n, k}^{U, T_{2}} \mathbf{H}_{n, k}^{B U}\left(\mathbf{H}_{n, k}^{B U}\right)^{\mathrm{H}}\left(\mathbf{R}_{n, k}^{U, T_{2}}\right)^{\mathrm{H}}$ and 447 $\mathbf{A}_{m}=\underline{\mathbf{H}}_{n, m, k}^{R U, T_{2}}\left(\underline{\mathbf{H}}_{n, m, k}^{R U, T_{2}}\right)^{\mathrm{H}}=\mathbf{R}_{n, k}^{U, T_{2}} \mathbf{H}_{n, m, k}^{R U}\left(\mathbf{H}_{n, m, k}^{R U}\right)^{\mathrm{H}}\left(\mathbf{R}_{n, k}^{U, T_{2}}\right)^{\mathrm{H}}, 448$ $\forall m$ are as small as possible. This design goal may be for- 449 malized as

$$
\begin{aligned}
\min _{\mathbf{R}_{n, k}^{U, T_{2}}} & \left\|\mathbf{H}_{n, k}^{B U}\left(\mathbf{H}_{n, k}^{B U}\right)^{\mathrm{H}}-\left(\mathbf{R}_{n, k}^{U, T_{2}}\right)^{-1} \boldsymbol{\Lambda}_{0}\left(\mathbf{R}_{n, k}^{U, T_{2}}\right)^{-\mathrm{H}}\right\|_{\mathrm{F}}^{2} \\
& +\sum_{m=1}^{M} \| \mathbf{H}_{n, m, k}^{R U}\left(\mathbf{H}_{n, m, k}^{R U}\right)^{\mathrm{H}} \\
& -\left(\mathbf{R}_{n, k}^{U, T_{2}}\right)^{-1} \boldsymbol{\Lambda}_{m}\left(\mathbf{R}_{n, k}^{U, T_{2}}\right)^{-\mathrm{H}} \|_{\mathrm{F}}^{2}
\end{aligned}
$$



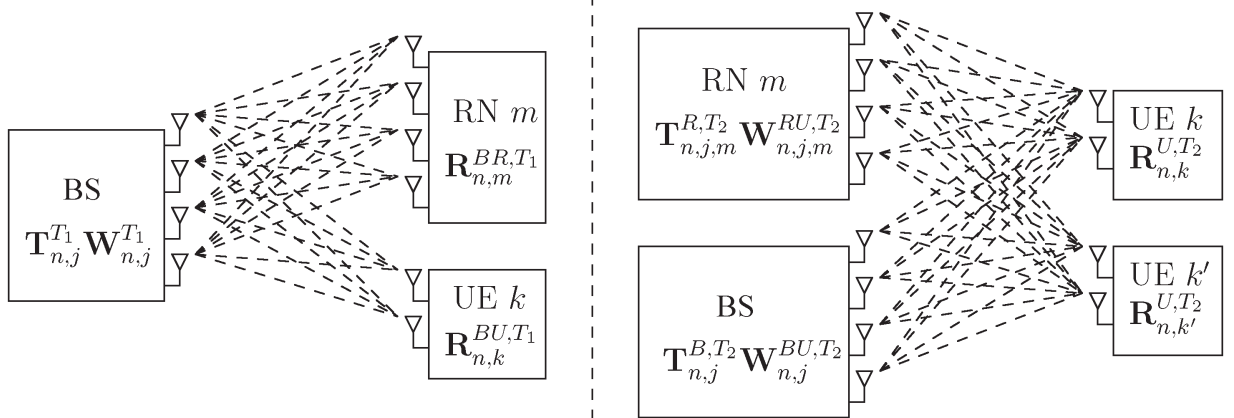

Fig. 3. A conceptual schematic of the transmit- and receive-BF matrices employed in the first and second transmission phases. In the first phase, the BS employs its ZFBF matrix to serve multiple data streams without interference. In the second phase, the BS and RNs employ separate ZFBF matrices to distributively avoid interference between the data streams being served.

451 where $\boldsymbol{\Lambda}_{0}$ and $\boldsymbol{\Lambda}_{m}$ are diagonal matrices containing the 452 diagonal elements of $\mathbf{A}_{0}$ and $\mathbf{A}_{m}$, respectively. Therefore, $453\left(\mathbf{R}_{n, k}^{U, T_{2}}\right)^{-1}$ is the jointly diagonalizing matrix [34], while $454 \mathbf{H}_{n, k}^{B U}\left(\mathbf{H}_{n, k}^{B U}\right)^{\mathrm{H}}$ and $\mathbf{H}_{n, m, k}^{R U}\left(\mathbf{H}_{n, m, k}^{R U}\right)^{\mathrm{H}}, \forall m$ are the matrices 455 to be diagonalized. Thus, the algorithm presented in [34] for 456 solving ${ }^{11}$ (2) may be invoked at UE $k$ for obtaining $\mathbf{R}_{n, k}^{U, T_{2}}$, 457 which may be further fed back to the BS and RNs. Hence, the $458 \mathrm{BS}$ and RNs do not have to share $\mathbf{H}_{n, k}^{B U}$ or $\mathbf{H}_{n, k}^{R U}$ via the wireless 459 channel and do not have to solve (2) again. As a result, we 460 accomplish the goal of creating effective channel matrices that 461 contain rows aiming to satisfy (1). Additionally, the columns 462 of $\mathbf{R}_{n, k}^{U, T_{2}}$ have been normalized so that the power assigned for 463 each SMC remains unaffected.

464 After obtaining the receive-BF matrix, the SMCs of the 465 transmissions to UE $k$ on subcarrier block $n$ are given by the 466 non-zero rows of the effective channel matrices $\underline{\mathbf{H}}_{n, k}^{B U, T_{2}}$ and $467 \underline{\mathbf{H}}_{n, m, k}^{R U, T_{2}}, \forall m$. Since the BS and the RNs act as distributed 468 broadcasters in the second phase, they are only capable of 469 employing separate ZFBF transmit matrices to ensure that 470 none of them imposes interference on the SMCs it does not 471 explicitly intend to serve. By employing one of the grouping 472 algorithms described in Section IV, the BS schedules ${ }^{12} Q_{j}^{T_{2}} \leq$ $473 \min \left[\min \left(N_{B}, N_{R}\right), \sum_{i=1}^{K} L_{i}^{B}+L_{i}^{R}\right]$ SMCs to serve simultane474 ously in the second phase, where $L_{i}^{B}$ and $L_{i}^{R}$ represent the 475 number of SMCs of UE $i$ served by the BS and by RNs in 476 this phase, respectively, where we have $L_{i}^{B}+L_{i}^{R} \leq N_{U}, L_{i}^{B} \leq$ $477 \min \left(N_{B}, N_{U}\right)$, and $L_{i}^{R} \leq \min \left(N_{R}, N_{U}\right)$. Note that since UE $i$ 478 may be simultaneously served both by the BS and by a RN 479 (each of them serves a fraction of UE $i$ 's SMCs), it is possible

\footnotetext{
${ }^{11}$ In fact, when there are only two matrices to diagonalize, say $\mathbf{A}_{0}$ and $\mathbf{A}_{1}$, the diagonalizing matrix may be obtained from the eigenvectors of $\mathbf{A}_{0}\left(\mathbf{A}_{1}\right)^{-1}$ [35]. This diagonalizing matrix is able to fully diagonalize both $\mathbf{A}_{0}$ and $\mathbf{A}_{1}$.

${ }^{12}$ To elaborate a little further, when computing its ZFBF transmit matrix, each transmitter (either the BS or a RN) must take into account an auxiliary SMC, which is also selected from the legitimate SMC candidates and is required for nulling the interference that this particular transmitter imposes on each selected information-bearing SMC of the other transmitters. Furthermore, each auxiliary SMC is employed by its corresponding transmitter to transmit several additional zeros that are padded to the normal data symbols. As a beneficial result, no interference is received at each UE from the transmitter that does not serve this particular UE. For more details of the SMC-based transmission in the second phase, please refer to Algorithm 1 described in Section IV-A.
}

that the summation of the respective number of UEs served ${ }^{13} 480$ by the BS and by RNs may be higher than $K$. Let us denote 481 the refined effective DL channel matrices, from the perspectives 482 of the BS and RN $m$, consisting of the $Q_{j}^{T_{2}}$ selected SMCs as 483 $\underline{\mathbf{H}}_{n, j}^{B, T_{2}} \in \mathbb{C}^{Q_{j}^{T_{2}} \times N_{B}}$ and $\underline{\mathbf{H}}_{n, j, m}^{R, T_{2}} \in \mathbb{C}^{Q_{j}^{T_{2}} \times N_{R}}$, respectively. Since 484 these are known to each transmitter, they may employ ZFBF 485 transmit matrices in the second phase, given by the right 486 inverses $\mathbf{T}_{n, j}^{B, T_{2}}=\left(\underline{\mathbf{H}}_{n, j}^{B, T_{2}}\right)^{\mathrm{H}} \cdot\left[\underline{\mathbf{H}}_{n, j}^{B, T_{2}}\left(\underline{\mathbf{H}}_{n, j}^{B, T_{2}}\right)^{\mathrm{H}^{-1}} \in \mathbb{C}^{N_{B} \times Q_{j}^{T_{2}}} 487\right.$ for the BS, and $\mathbf{T}_{n, j, m}^{R, T_{2}}=\left(\underline{\mathbf{H}}_{n, j, m}^{R, T_{2}}\right)^{\mathrm{H}} \cdot\left[\underline{\mathbf{H}}_{n, j, m}^{R, T_{2}}\left(\underline{\mathbf{H}}_{n, j, m}^{R, T_{2}}\right)^{\mathrm{H}^{-1}} \in 488\right.$ $\mathbb{C}^{N_{R} \times Q_{j}^{T_{2}}}$ for RN $m$. Similar to the first transmission phase, 489 these ZFBF transmit matrices are normalized by $\mathbf{W}_{n, j}^{B U, T_{2}}$ and 490 $\mathbf{W}_{n, j, m}^{R U, T_{2}}$, respectively, to ensure that each SMC transmission 491 is initially set to unit power. Upon obtaining the selected 492 SMCs, we denote the effective CNRs in the second trans- 493 mission phase as $G_{n, j, e_{2}}^{B U, T_{2}}=\left|w_{n, j, e_{2}}^{B U, T_{2}}\right|^{2} / \Delta \gamma N_{0} W$ and $G_{n, j, e}^{R U, T_{2}}=494$ $\left|w_{n, j, e}^{R U, T_{2}}\right|^{2} / \Delta \gamma N_{0} W$, where $w_{n, j, e_{2}}^{B U, T_{2}}$ and $w_{n, j, e}^{R U, T_{2}}$ are the diago- 495 nal elements in $\mathbf{W}_{n, j}^{B U, T_{2}}$ and $\mathbf{W}_{n, j, \mathcal{M}(e)}^{R U, T_{2}}$, respectively, and the 496 subscript $\mathcal{M}(e)$ has been defined in Section III-A. To elabo- 497 rate, for a second-phase BS-to-UE link, $w_{n, j, e_{2}}^{B U, T_{2}}$ corresponds 498 to SMC group $j$ and subcarrier block $n$, while the subscript 499 $e_{2} \in\left\{0, \ldots, \min \left[N_{B}, K \cdot \min \left(N_{B}, N_{U}\right)\right]\right\}$ is employed for fur- 500 ther distinguishing the multiple selected SMCs associated with 501 UEs from the BS. Similarly, $w_{n, j, e}^{R U, T_{2}}$, which also corresponds 502 to SMC group $j$ and subcarrier block $n$, is associated with 503 the second-phase RN-to-UE link between RN $\mathcal{M}(e)$ and the 504 particular UE of SMC-pair $e$.

For more explicit clarity, a schematic of the transmit and re- 506 ceive beamforming matrices in the first and second transmission 507 phases is presented in Fig. 3.

\section{Achievable Spectral Efficiency and Energy} Spectral Efficiency

For the sake of convenience, let us first denote the transmit 511 power allocation policy as $\mathcal{P}$, which is a set composed by all 512 transmit power control variables invoked at the BS and/or RNs 513

\footnotetext{
${ }^{13}$ If at least one SMC of a UE is served by the BS (or a RN), we say that this UE is served by the BS (or the RN).
} 
514 in both transmission phases. Since receive-BF is employed in 515 conjunction with ZFBF, each SMC transmission may be viewed 516 as a single-input-single-output (SISO) link. Therefore, on the 517 direct links, the receiver's SNR at UE $k$ corresponding to SMCs $518 e_{1}$ and $e_{2}$ may be expressed as $\Gamma_{n, j, e_{1}}^{B U, T_{1}}(\mathcal{P})=G_{n, j, e_{1}}^{B U, T_{1}} P_{n, j, e_{1}}^{B U, T_{1}}$ 519 and $\Gamma_{n, j, e_{2}}^{B U, T_{2}}(\mathcal{P})=G_{n, j, e_{2}}^{B U, T_{2}} P_{n, j, e_{2}}^{B U, T_{2}}$ for the first and second 520 transmission phases, respectively. The scalar variables $P_{n, j, e_{1}}^{B U, T_{1}}$ 521 and $P_{n, j, e_{2}}^{B U, T_{2}}$, which are elements of $\mathcal{P}$, determine the transmit 522 power values for SMCs $e_{1}$ and $e_{2}$ on the direct links. As a result, 523 the achievable instantaneous SE of the direct links is given by $524 C_{n, j, e_{1}}^{B U, T_{1}}(\mathcal{P})=(1 / 2) \log _{2}\left(1+\Gamma_{n, j, e_{1}}^{B U, T_{1}}(\mathcal{P})\right)$ and $C_{n, j, e_{2}}^{B U, T_{2}}(\mathcal{P})=$ $525(1 / 2) \log _{2}\left(1+\Gamma_{n, j, e_{2}}^{B U, T_{2}}(\mathcal{P})\right)$, which are normalized both by 526 time and by frequency to give units of [bits/sec/Hz]. The factor 527 of $1 / 2$ accounts for the fact that the transmission period is split 528 into two phases.

529 Similarly, for the SMC-pair $e$ of the DF relaying links, the $530 \mathrm{SNR}$ at $\mathrm{RN} \mathcal{M}(e)$ in the first transmission phase is given 531 by $\Gamma_{n, j, e}^{B R, T_{1}}(\mathcal{P})=G_{n, j, e}^{B R, T_{1}} P_{n, j, e}^{B R, T_{1}}$ and the SNR at UE $k$ in 532 the second transmission phase is formulated as $\Gamma_{n, j, e}^{R U, T_{2}}(\mathcal{P})=$ $533 G_{n, j, e}^{R U, T_{2}} P_{n, j, e}^{R U, T_{2}}$. Additionally, $P_{n, j, e}^{B R, T_{1}}$ and $P_{n, j, e}^{R U, T_{2}}$ are also 534 elements of $\mathcal{P}$. Since the RNs employ the DF protocol, the 535 achievable SE is limited by the weaker of the two RN-related 536 links [29] and is given by $C_{n, j, e}^{B R U}(\mathcal{P})=\min \left[(1 / 2) \log _{2}(1+\right.$ $\left.\left.537 \Gamma_{n, j, e}^{B R, T_{1}}(\mathcal{P})\right),(1 / 2) \log _{2}\left(1+\Gamma_{n, j, e}^{R U, T_{2}}(\mathcal{P})\right)\right]$.

538 Let us now introduce the SMC group selection variable $539 s_{n, j} \in\{0,1\}$, which indicates that SMC group $j$, as introduced 540 in Sections III-A and III-B, is selected for subcarrier block $541 n$, when $s_{n, j}=1$, and $s_{n, j}=0$ otherwise. All SMC group 542 selection variables are scalars and are collected into a set 543 denoted by $\mathcal{S}$. Once again, we emphasize that $\mathcal{G}_{n}$ denotes the 544 set of possible SMC groups for subcarrier block $n$. Thus, the 545 total achieved SE is given by (3) (see equation at the bottom of 546 the page), where $\mathcal{E}_{n, j}$ is the set of SMCs in the selected group $j$ 547 on subcarrier block $n$.

548 In this work, we adopt the energy dissipation model pre549 sented in [36], where the total energy dissipation of the system 550 is assumed to be dependent on several factors, including the 551 number of TAs, the energy dissipation of the RF and baseband 552 circuits, and the efficiencies of the power amplifier, feeder 553 cables, cooling system, mains power supply, and converters. 554 For the sake of simplicity, the total energy dissipation as 555 presented in [36] has been partitioned into a fixed term, and a term that varies with the transmission powers. Thus, the 556 energy dissipation of the system may be characterized by (4) 557 (see equation at the bottom of the page), where $P_{C}^{B}$ and $P_{C}^{R} 558$ represent the fixed energy dissipation of each BS and each RN, 559 respectively, while $\xi^{B}>1$ and $\xi^{R}>1$ are the energy dissipa- 560 tion multipliers of the transmit powers for the BS and the RNs, 561 respectively. The effect of multiple transmit antennas on the 562 total energy dissipation has been included in the terms $P_{C}^{B}, P_{C}^{R}, 563$ $\xi^{B}$, and $\xi^{R}$.

Finally, the ESE of the system is expressed as

$$
\eta_{E}(\mathcal{P}, \mathcal{S})=\frac{C_{T}(\mathcal{P}, \mathcal{S})}{P_{T}(\mathcal{P}, \mathcal{S})}
$$

The objective of this paper is to maximize (5) by appropriately 566 optimizing $\mathcal{P}$ and $\mathcal{S}$.

\section{Semi-Orthogonal Grouping Algorithms}

As described in Section II, the BS has to choose $Q_{j}^{T_{1}}$ and 569 $Q_{j}^{T_{2}}$ SMCs for the first and second transmission phases, re- 570 spectively. These selected SMCs collectively form the SMC 571 group $j$. Since the system supports both direct and relaying 572 links, the grouping algorithms described in [7], [8], which were 573 designed for MIMO systems dispensing with relays, may not be 574 directly applied. Instead, we propose a pair of viable grouping 575 algorithms, namely the exhaustive search-based grouping algo- 576 rithm (ESGA), and the orthogonal component-based grouping 577 algorithm (OCGA).

578

Note that because there are multiple distributed transmit- 579 ters in the second transmission phase, each UE designs its 580 receive-BF matrix by jointly considering all the MIMO chan- 581 nel matrices associated with it, as described in Section III-B. 582 However, before applying this method, we have to determine 583 which particular transmitters (out of the BS and RNs) should 584 actively transmit in the second transmission phase based on the 585 results of SMC selection. Note that it is possible that the SMC 586 candidates obtained may lead to higher effective CNRs when 587 a subset of the transmitters are inactive. On the one hand, an 588 additional effect of only activating a subset of transmitters is the 589 reduced number of SMC candidates, which might in turn result 590 in a reduced number of qualified SMCs that satisfy the semi- 591 orthogonality criterion considered. As a result, the achievable 592 spatial multiplexing gain and SE might be degraded. On the 593

$$
C_{T}(\mathcal{P}, \mathcal{S})=\sum_{n=1}^{N} \sum_{j \in \mathcal{G}_{n}} s_{n, j}\left[\sum_{e_{1} \in \mathcal{E}_{n, j}} C_{n, j, e_{1}}^{B U, T_{1}}(\mathcal{P})+\sum_{e_{2} \in \mathcal{E}_{n, j}} C_{n, j, e_{2}}^{B U, T_{2}}(\mathcal{P})+\sum_{e \in \mathcal{E}_{n, j}} C_{n, j, e}^{B R U}(\mathcal{P})\right]
$$

$$
P_{T}(\mathcal{P}, \mathcal{S})=\left(P_{C}^{B}+M \cdot P_{C}^{R}\right)+\frac{1}{2} \sum_{n=1}^{N} \sum_{j \in \mathcal{G}_{n}} s_{n, j} \times\left[\xi^{B}\left(\sum_{e_{1} \in \mathcal{E}_{n, j}} P_{n, j, e_{1}}^{B U, T_{1}}+\sum_{e_{2} \in \mathcal{E}_{n, j}} P_{n, j, e_{2}}^{B U, T_{2}}\right)+\sum_{e \in \mathcal{E}_{n, j}}\left(\xi^{B} P_{n, j, e}^{B R, T_{1}}+\xi^{R} P_{n, j, e}^{R U, T_{2}}\right)\right]
$$


594 other hand, this SE-reduction effect may be counteracted by 595 the improved CNRs gleaned from the fact that it is easier to 596 generate SMCs that can satisfy a stricter semi-orthogonality 597 criterion, specified by a smaller value of $\alpha$, when the number 598 of transmitters is lower. For example, in the scenarios where 599 only one or two active transmitters are selected, the UEs can 600 employ receive-BF matrices that create effective DL channel 601 matrices containing completely orthogonal rows by using the 602 SVD or the exact diagonalization method (see Footnote ${ }^{11}$ ), 603 respectively. To account for this dilemma, for the second trans604 mission phase, the proposed grouping algorithms evaluate a full 605 list of SMCs, which consists of the SMCs obtained from the $6062^{M+1}-1$ possible combinations of active transmitters (the BS 607 and $M$ RNs, while ignoring the case when there are no active 608 transmitters). Compared to using a smaller list of SMCs, using 609 a full list of SMCs ensures that achieving a lower-bound SE 610 is always guaranteed, while a higher SE can only be obtained 611 upon increasing the number of transmitters in the system.

\section{A. SMC Checking Algorithm}

613 Both grouping algorithms must evaluate a particular SMC 614 before it may be included into the SMC group to be generated. 615 This evaluation process is depicted in Algorithm 1. More 616 specifically, the algorithm identifies the transmitters associated 617 with each SMC of the current SMC group, denoted by $\mathcal{E}_{n, j}$, 618 in lines 7 to 17 . The transmitter associated with the candidate $619 \mathrm{SMC}, e_{c}$, is identified in lines 18 to 28 . Additionally, as briefly 620 pointed out in Footnote ${ }^{12}$, for an active transmitter, if the 621 candidate SMC is associated with a transmission in the second 622 phase, then the auxiliary SMCs, $e^{\varnothing}$ and $e_{m}^{\varnothing}$, are included for 623 the other active transmitters in lines 23, 26 and 27, to ensure 624 that these potentially interfering transmitters do not impose 625 interference on the candidate SMC. ${ }^{14}$ Note that $e^{\varnothing}$ and $e_{m}^{\varnothing}$ 626 represent auxiliary SMCs invoked by the BS and RNs, respec627 tively. Having determined the transmitters associated with the 628 SMCs, the algorithm checks that the SMCs associated with the 629 same transmitter satisfy the semi-orthogonality criterion of (1) 630 having parameter $\alpha$ in lines 20, 29, and 30. Furthermore, the 631 algorithm ensures that the inclusion of the candidate SMC does 632 not force any of the transmitters to transmit over its maximum 633 number of transmit dimensions, as depicted in lines 29 and 30. 634 Meanwhile, each UE should not receive more than its maxi635 mum number of receive dimensions, which is accomplished in 636 lines 12, 32, and 33. Finally, the maximum achievable spatial 637 multiplexing gain should not be exceeded in either the first or 638 second phase, which is ensured by lines 35 and 36 . If all of these 639 checks are successful, the algorithm exits with a true condition 640 in line 37.

\footnotetext{
${ }^{14}$ For distributed transmitters encountered in the second transmission phase, it is not feasible to design a single ZFBF transmit matrix as we did for the BS in the first transmission phase. For the second transmission phase, when $N_{B} \leq$ $N_{U}$ and $N_{R} \leq N_{U}$, each SMC is associated with a single receive antenna. Consider this case as an example, when the BS is transmitting on a SMC to a particular receive antenna of a UE, an active RN may be transmitting zeros on an auxiliary SMC, which is also selected from the legitimate SMC candidates, to the same receive antenna of that UE. As a beneficial result of this strategy, for each transmitter, the interference imposed by other active transmitters is nulled.
}

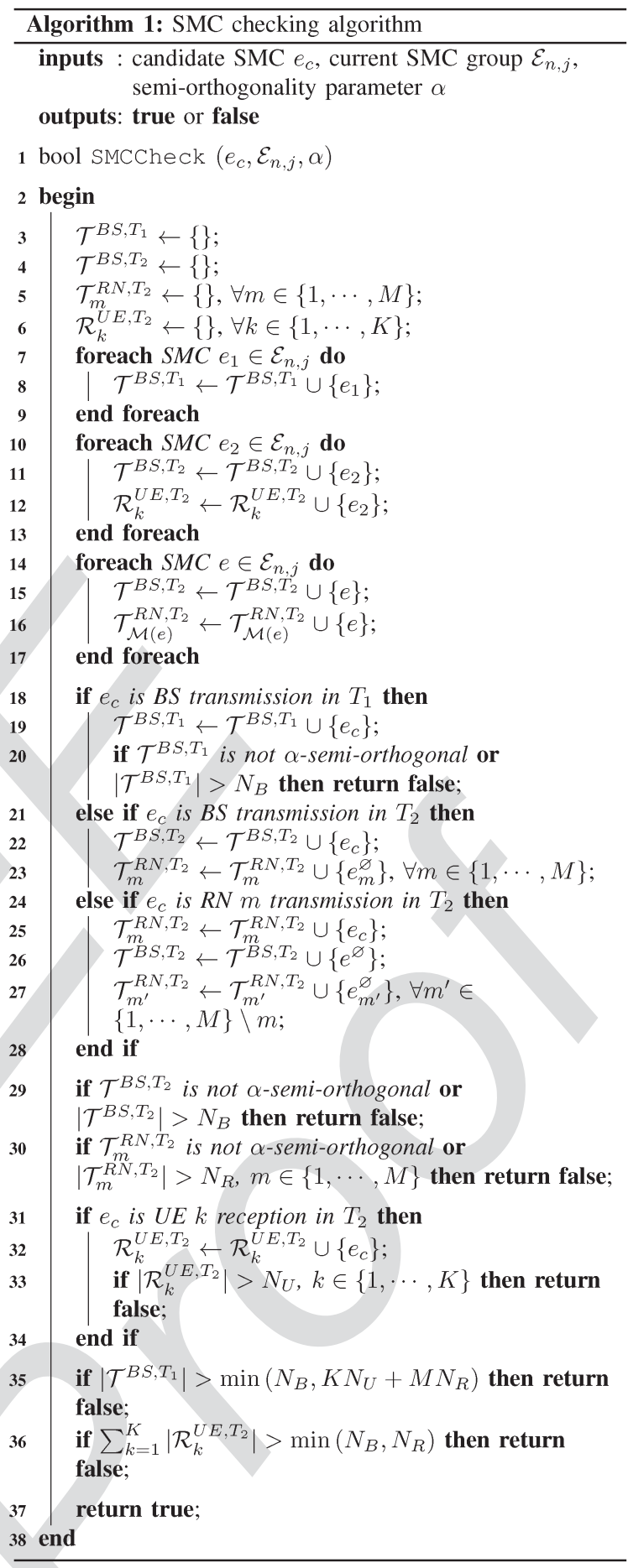

\section{B. ESGA and OCGA}

We present our first grouping method in Algorithm 2. Simply 642 put, the ESGA recursively creates new SMC groups by exhaus- 643 tively searching through all the possible combinations of SMCs 644 and including those that pass the SMC checking algorithm. 645 To elaborate, in the loop ranging from line 3 to line 9, the 646 algorithm searches through all the possible SMCs associated 647 with subcarrier block $n$, which are collectively denoted by $\mathcal{E}_{n} 648$ and satisfy $e_{c} \in \mathcal{E}_{n}$. The specific SMCs that satisfy the checks 649 performed in line 4 are appended to the current SMC group in 650 line 5, and the resultant updated SMC group $\mathcal{E}_{n, j^{\prime}}^{\prime}$ is appended 651 to the set of SMC groups obtained for subcarrier block $n$ in line 652 
6536 . Additionally, $\mathcal{E}_{n, j^{\prime}}^{\prime}$ is used recursively in line 7 for filling this 654 group and for forming new groups. The computational com655 plexity of ESGA is dependent on the number of SMCs which 656 are semi-orthogonal to each other. The worst-case complexity 657 is obtained when every SMC satisfies the checks performed in 658 line 4, leading to a time-complexity (in terms of the number of 659 SMC groups generated) upper-bounded (not necessarily tight) 660 by $\mathcal{O}\left(\sum_{n=1}^{N}\left|\mathcal{E}_{n}\right|^{\theta}\right)$, where

$$
\begin{aligned}
\theta=\min \left[N_{B}, K\right. & \left.\cdot \min \left(N_{B}, N_{U}\right)+M \cdot \min \left(N_{B}, N_{R}\right)\right] \\
& +\min \left[\min \left(N_{B}, N_{R}\right), \sum_{i=1}^{K} L_{i}^{B}+L_{i}^{R}\right] .
\end{aligned}
$$

661 In other words, each subcarrier block may be treated indepen662 dently. For each subcarrier block, $\left|\mathcal{E}_{n}\right|$ SMCs must be checked 663 until the maximum multiplexing gain in both the first and 664 second phases has been attained.

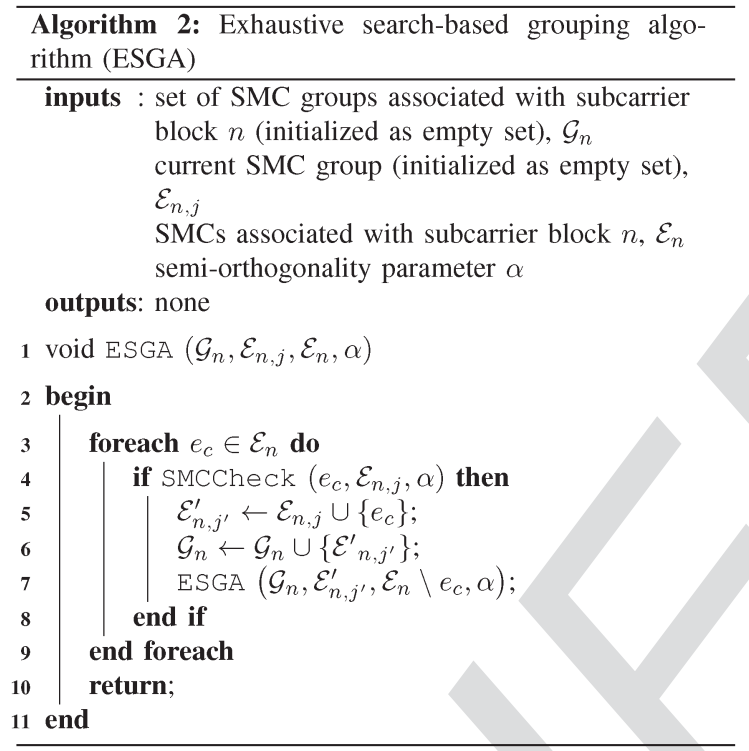

665 The second algorithm, OCGA, is presented in Algorithm 3, 666 which aims to be a lower complexity alternative to ESGA. The 667 OCGA commences by creating a SMC candidate set $\mathcal{E}_{c}$, whose 668 elements satisfy the checks performed in Algorithm 1, in lines 4 669 to 6 . More specifically, if the current SMC group $\mathcal{E}_{n, j}$ is empty, 670 the algorithm can simply create a new SMC group containing 671 only the candidate SMC that has passed the SMC checks of 672 Algorithm 1 in lines 7 to 10 . If the SMC group is not empty, the 673 algorithm adds to it the particular SMC candidate that results 674 in the highest norm of the orthogonal component (NOC), via 675 the Gram-Schmidt procedure [7], [8], in line 20. This process 676 is repeated until the maximum multiplexing gain in both the 677 first and second phases has been attained. When comparing 678 the NOCs obtained for the relaying links, the minimum of the 679 NOCs obtained from the BS-to-RN and RN-to-UE SMCs is 680 used. This is because the information conveyed on the relaying 681 link is limited by the weaker of the two transmissions, which 682 is reflected in the effective channel gains quantified by these 683 norms. If no SMCs satisfy the checks of line 6, the current 684 SMC group is complete, and it is appended to the current set of 685 SMC groups in line 18 . Since new groups are only created when 686 the current SMC group is empty, this algorithm results in much fewer groups than ESGA. The algorithmic time-complexity is 687 given by $\mathcal{O}\left(\sum_{n=1}^{N}\left|\mathcal{E}_{n}\right|\right)$ as a single group is created for each 688 initially-selected SMC.

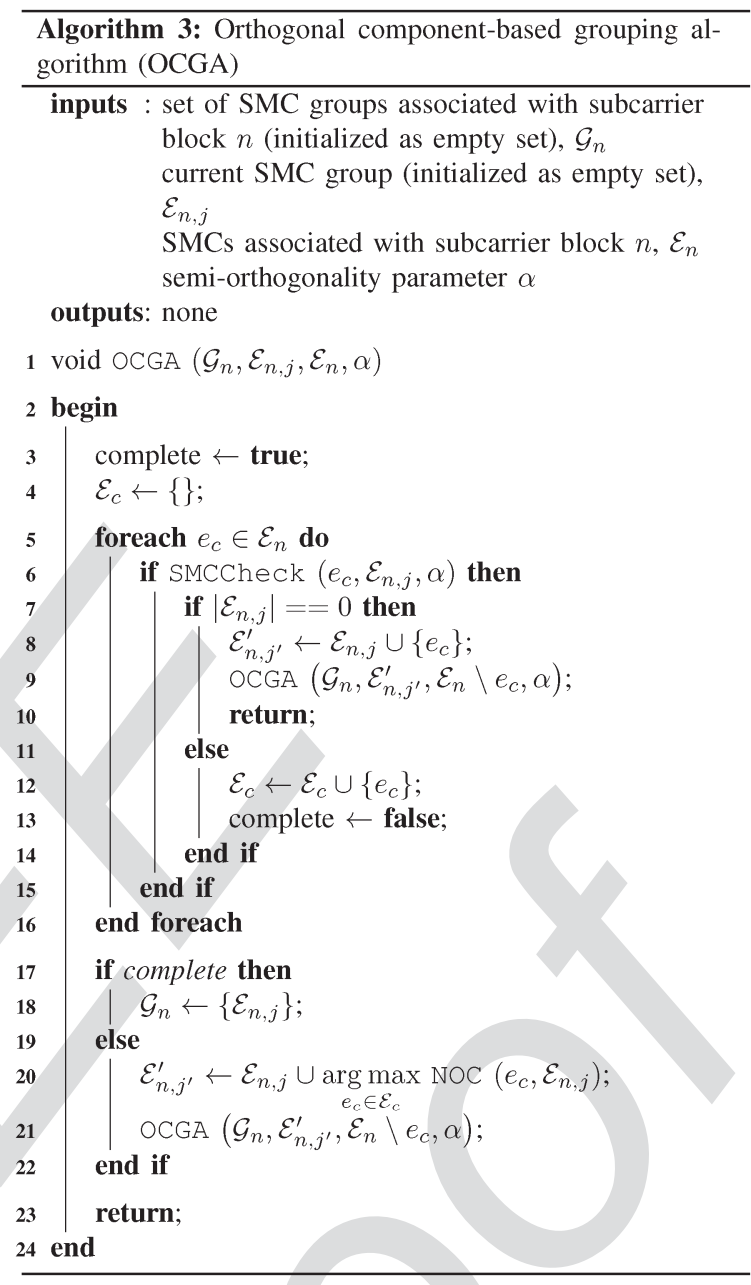

Both grouping algorithms may be initialized with an empty 690 SMC group, $\mathcal{E}_{n, j} \leftarrow\{\}$, and an empty set of SMC groups, 691 $\mathcal{G}_{n} \leftarrow\{\}$, so that they recursively create and fill SMC groups 692 according to their criteria. Additionally, a final step is per- 693 formed to remove the specific groups, which result in effective 694 channel gains that are less than or equal to that of another group, 695 while having the same transmitters. Therefore, this final step 696 does not reduce the attainable SE or ESE, but reduces the num- 697 ber of possible groups, thus alleviating the computational com- 698 plexity imposed by the optimization algorithms of Section V-C. 699

\section{SEM/ESEM PRoblem Formulation AND SOLUTiOn 700}

Having obtained the set of SMC groups $\mathcal{G}_{n}$ for each subcar- 701 rier block $n$, in this section our aim is to find the optimum power 702 variables contained in $\mathcal{P}$ and optimum SMC-group selection 703 variables contained in $\mathcal{S}$, so that (5) is maximized. We com- 704 mence by formulating the problem of maximizing the SE of the 705 system as (7)-(13) (see equation at the bottom of the next page). 706

To elaborate, (7) represents the sum SE of the system, which 707 is formulated in more detail as (3). The constraints (9)-(11) 708 ensure that the maximum instantaneous transmission power 709 constraint is never exceeded in either of the two transmission 710 
711 phases for the BS and the RNs, while the constraints (8) and 712 (12) ensure that only a single SMC group is selected for each 713 subcarrier block. Finally, (13) restricts the power variables to 714 be non-negative.

\section{A. Relaxed SEM Problem}

716 Although the constraint (13) is affine (hence convex) in 717 the optimization variables contained in $\mathcal{P},(8)-(11)$ are non718 convex [32], because (12) imposes a binary constraint on the 719 problem. Furthermore, the objective function given by (7) is 720 not concave, since it is dependent on the binary variables given 721 by $\mathcal{S}$. Thus, (7)-(13) may be classified as a mixed-integer non722 linear programming (MINLP) problem, which may be solved 723 using branch-and-bound methods [37]. However, these meth724 ods typically incur a computational complexity that increases 725 exponentially in the number of discrete variables, which is 726 undesirable for practical implementations. To circumvent this 727 initial setback, we introduce the following auxiliary variables

$$
\begin{aligned}
\widetilde{P}_{n, j, e_{1}}^{B U, T_{1}}=P_{n, j, e_{1}}^{B U, T_{1}} \widetilde{s}_{n, j}, & \forall n, j, e_{1}, \\
\widetilde{P}_{n, j, e}^{B R, T_{1}}=P_{n, j, e}^{B R, T_{1}} \widetilde{s}_{n, j}, & \forall n, j, e, \\
\widetilde{P}_{n, j, e_{2}}^{B U, T_{2}}=P_{n, j, e_{2}}^{B U, T_{2}} \widetilde{s}_{n, j}, & \forall n, j, e_{2}, \\
\widetilde{P}_{n, j, e}^{R U, T_{2}}=P_{n, j, e}^{R U, T_{2}} \widetilde{s}_{n, j}, & \forall n, j, e, \\
\widetilde{C}_{n, j, e_{1}}^{B U, T_{1}}, \quad \widetilde{C}_{n, j, e_{2}}^{B U, T_{2}} \text { and } \widetilde{C}_{n, j, e}^{B R U}, & \forall n, j, e_{1}, e_{2}, e,
\end{aligned}
$$

728 where we have relaxed ${ }^{15}$ the binary constraint of (12) to give

$$
0 \leq \widetilde{s}_{n, j} \leq 1, \quad \forall n, j
$$

${ }^{15}$ In [38], such a relaxation results in a time-sharing solution regarding each subcarrier. In this work, this relaxation may be viewed as time-sharing of each subcarrier block, as multiple SMC groups can then occupy a fraction of each subcarrier block in time. Naturally, the relaxation means that we do not accurately solve the original problem of (7)-(13). However, as shown in [17], [21], [27], the solution to the original problem is still obtained with high probability when using the dual decomposition method on the relaxed problem (as in this work) as the number of subcarriers tends to infinity. It was shown that 8 subcarriers is sufficient for this to be true in the context of [39], while we have shown that 2 subcarriers is sufficient in the context of [17]. so that we may write (7)-(13) in the hypograph problem [32] 729 form given by (20)-(30), ${ }^{16}$ (see equation at the bottom of the 730 page), where $\widetilde{\mathcal{C}}, \widetilde{\mathcal{P}}$ and $\widetilde{\mathcal{S}}$ indicate the variable-sets containing 731 their associated auxiliary variables.

732

It can be seen that the objective function of (7) has been 733 replaced by (20) using the auxiliary rate variables given in 734 (18), and by introducing the hypograph constraints (21)-(24). ${ }^{17} 735$ These additional constraints ensure that the feasible auxiliary 736 rate variables do not exceed their counterparts calculated on 737 each link before using relaxation. As a result, the sum rate given 738 by (20) invoking the feasible auxiliary rate variables does not 739 exceed the sum rate given by (3) either.

As our next step, we prove that the problem described by 741 (20)-(30) is a concave programming problem. Clearly, (20) is 742 affine, hence concave, while (25)-(30) are all affine, and hence 743 convex. Therefore, what remains is to show that constraints 744 (21)-(24) are convex as well. These remaining constraints may 745 be written in the form of

$$
C-\frac{s}{2} \log _{2}\left(1+\frac{G P}{s}\right) \leq 0,
$$

where $s, P$ and $C$ are the decision variables. It may be 747 readily verified that $(1+G P)$ is affine and hence concave. 748 Thus, $\log _{2}(1+G P)$ is concave, since $\log _{2}(\cdot)$ is concave 749 and non-decreasing as a function of its argument. The func- 750 tion $s \log _{2}(1+(G P / s))$ is a perspective transformation ${ }^{18}[32] 751$

${ }^{16}$ Writing the original optimization problem in the hypograph form of (20)-(30) means that minimum per-link or system-wide SE constraints may be readily introduced. However, minimum SE constraints are not considered in this paper as our goal is to find the maximum SE/ESE solutions, which may not be equivalent to the solutions obtained when satisfying minimum SE constraints.

${ }^{17}$ Note that obtaining separate constraints for the first- and second-phase power control variables associated with the relayed transmission is made possible using the DF protocol. This then allowed us to readily derive the optimal power control variables as the decoupled water-filling solutions in Section V-C.1.

${ }^{18}$ Strictly speaking, the perspective transformation also requires that $s>0$. However, convexity is also preserved for the situation when $s=0$ as proven in [40].

$$
\begin{aligned}
\underset{\mathcal{P}, \mathcal{S}}{\operatorname{maximize}} & C_{T}(\mathcal{P}, \mathcal{S}) \\
\text { subject to } & \sum_{j \in \mathcal{G}_{n}} s_{n, j} \leq 1, \quad \forall n, \\
& \sum_{i=1}^{N} \sum_{j \in \mathcal{G}_{n}} s_{n, j}\left[\sum_{e_{1} \in \mathcal{E}_{n, j}} P_{n, j, e_{1}}^{B U, T_{1}}+\sum_{e \in \mathcal{E}_{n, j}} P_{n, j, e}^{B R, T_{1}}\right] \leq P_{\text {max }}^{B}, \\
& \sum_{i=1}^{N} \sum_{j \in \mathcal{G}_{n}} s_{n, j} \sum_{e_{2} \in \mathcal{E}_{n, j}} P_{n, j, e_{2}}^{B U, T_{2}} \leq P_{\max }^{B}, \\
& \sum_{i=1}^{N} \sum_{j \in \mathcal{G}_{n}} s_{n, j} \sum_{\substack{e \in \mathcal{E}_{n, j} \\
\mathcal{M}(e)=m}} P_{n, j, e}^{R U, T_{2}} \leq P_{\max }^{R}, \quad \forall m, \\
& s_{n, j} \in\{0,1\}, n_{n, j,}, \quad \forall n, j, e_{1}, e_{2}, e \\
& P_{n, j, e_{1}}^{B U, T_{1}}, P_{n, j, e}^{B R, T_{1}}, P_{n, j, e_{2}}^{B U, T_{2}}, P_{n, j, e}^{R U, T_{2}} \geq 0, \quad \forall
\end{aligned}
$$


752 of $\log _{2}(1+G P)$, which preserves concavity. Finally, $C-$ $753(s / 2) \log _{2}(1+(G P / s))$ is convex, since it is the sum of two 754 convex functions. Since (31) is convex, it is clear that con755 straints (21)-(24) are convex, and so (20)-(30) is a concave 756 programming problem, whose solution algorithm is presented 757 in Section V-C.

\section{B. ESEM Problem}

759 The ESE objective function, given by (32) (see equation at 760 the bottom of the page), is formed by dividing the objective 761 function (20) by $P_{T}(\widetilde{\mathcal{P}}, \widetilde{\mathcal{S}})$, which is obtained by substituting 762 (14)-(17) into (4) and introducing the relaxed variables $\widetilde{s}_{n, j}$.

763 The objective function (32) is a linear-fractional function, 764 since it is a ratio of two affine functions. Thus the ESEM prob- lem can be solved using the Charnes-Cooper transformation of 765 [24], as given by

$$
\begin{array}{rlrl}
\widehat{C}_{n, j, e_{1}}^{B U, T_{1}} & =\widetilde{C}_{n, j, e_{1}}^{B U, T_{1}} t, \quad \forall n, j, e_{1}, \\
\widehat{C}_{n, j, e_{2}}^{B U, T_{2}} & =\widetilde{C}_{n, j, e_{2}}^{B U, T_{2}} t, \quad \forall n, j, e_{2}, \\
\widehat{C}_{n, j, e}^{B R U} & =\widetilde{C}_{n, j, e}^{B R U} t, & \forall n, j, e, \\
\widehat{P}_{n, j, e_{1}}^{B U, T_{1}} & =\widetilde{P}_{n, j, e_{1}}^{B U, T_{1}} t, & \forall n, j, e_{1}, \\
\widehat{P}_{n, j, e_{2}}^{B U, T_{2}} & =\widetilde{P}_{n, j, e_{2}}^{B U, T_{2}} t, \quad \forall n, j, e_{2}, \\
\widehat{P}_{n, j, e}^{B R, T_{1}} & =\widetilde{P}_{n, j, e}^{B R, T_{1}} t, \quad \forall n, j, e, \\
\widehat{P}_{n, j, e}^{R U, T_{2}} & =\widetilde{P}_{n, j, e}^{R U, T_{2}} t, \quad \forall n, j, e, \\
\widehat{s}_{n, j} & =\widetilde{s}_{n, j} t, \quad \forall n, j,
\end{array}
$$

$$
\begin{aligned}
& \underset{\widetilde{\mathcal{C},}, \widetilde{\mathcal{P}}, \widetilde{\mathcal{S}}}{\operatorname{maximize}} \sum_{i=1}^{N} \sum_{j \in \mathcal{G}_{n}}\left[\sum_{e_{1} \in \mathcal{E}_{n, j}} \widetilde{C}_{n, j, e_{1}}^{B U, T_{1}}+\sum_{e_{2} \in \mathcal{E}_{n, j}} \widetilde{C}_{n, j, e_{2}}^{B U, T_{2}}\right]+\left[\sum_{e \in \mathcal{E}_{n, j}} \widetilde{C}_{n, j, e}^{B R U}\right] \\
& \text { subject to } \frac{\widetilde{s}_{n, j}}{2} \log _{2}\left(1+\frac{G_{n, j, e_{1}}^{B U, T_{1}} \widetilde{P}_{n, j, e_{1}}^{B U, T_{1}}}{\widetilde{s}_{n, j}}\right) \geq \widetilde{C}_{n, j, e_{1}}^{B U, T_{1}}, \quad \forall n, j, e_{1} \text {, } \\
& \frac{\widetilde{s}_{n, j}}{2} \log _{2}\left(1+\frac{G_{n, j, e_{2}}^{B U, T_{2}} \widetilde{P}_{n, j, e_{2}}^{B U, T_{2}}}{\widetilde{s}_{n, j}}\right) \geq \widetilde{C}_{n, j, e_{2}}^{B U, T_{2}}, \quad \forall n, j, e_{2}, \\
& \frac{\widetilde{s}_{n, j}}{2} \log _{2}\left(1+\frac{G_{n, j, e}^{B R, T_{1}} \widetilde{P}_{n, j, e}^{B R, T_{1}}}{\widetilde{s}_{n, j}}\right) \geq \widetilde{C}_{n, j, e}^{B R U}, \quad \forall n, j, e, \\
& \frac{\widetilde{s}_{n, j}}{2} \log _{2}\left(1+\frac{G_{n, j, e}^{R U, T_{2}} \widetilde{P}_{n, j, e}^{R U, T_{2}}}{\widetilde{s}_{n, j}}\right) \geq \widetilde{C}_{n, j, e}^{B R U}, \quad \forall n, j, e, \\
& \sum_{j \in \mathcal{G}_{n}} \widetilde{s}_{n, j} \leq 1, \quad \forall n, \\
& \sum_{i=1}^{N} \sum_{j \in \mathcal{G}_{n}}\left[\sum_{e_{1} \in \mathcal{E}_{n, j}} \widetilde{P}_{n, j, e_{1}}^{B U, T_{1}}+\sum_{e \in \mathcal{E}_{n, j}} \widetilde{P}_{n, j, e}^{B R, T_{1}}\right] \leq P_{\max }^{B}, \\
& \sum_{i=1}^{N} \sum_{j \in \mathcal{G}_{n}} \sum_{e_{2} \in \mathcal{E}_{n, j}} \widetilde{P}_{n, j, e_{2}}^{B U, T_{2}} \leq P_{\text {max }}^{B} \\
& \sum_{i=1}^{N} \sum_{j \in \mathcal{G}_{n}} \sum_{\substack{e \in \mathcal{E}_{n, j} \\
\mathcal{M}^{(e)=m}}} \widetilde{P}_{n, j, e}^{R U, T_{2}} \leq P_{\max }^{R}, \quad \forall m, \\
& 0 \leq \widetilde{s}_{n, j} \leq 1, \quad \forall n, j, \\
& \widetilde{P}_{n, j, e_{1}}^{B U, T_{1}}, \widetilde{P}_{n, j, e}^{B R, T_{1}}, \widetilde{P}_{n, j, e_{2}}^{B U, T_{2}}, \widetilde{P}_{n, j, e}^{R U, T_{2}} \geq 0, \quad \forall n, j, e_{1}, e_{2}, e
\end{aligned}
$$

$$
\frac{\sum_{i=1}^{N} \sum_{j \in \mathcal{G}_{n}}\left[\sum_{e_{1} \in \mathcal{E}_{n, j}} \widetilde{C}_{n, j, e_{1}}^{B U, T_{1}}+\sum_{e_{2} \in \mathcal{E}_{n, j}} \widetilde{C}_{n, j, e_{2}}^{B U, T_{2}}\right]+\left[\sum_{e \in \mathcal{E}_{n, j}} \widetilde{C}_{n, j, e}^{B R U}\right]}{\left(P_{C}^{B}+M \cdot P_{C}^{R}\right)+\frac{1}{2} \sum_{n=1}^{N} \sum_{j \in \mathcal{G}_{n}}\left[\xi^{B}\left(\sum_{e_{1} \in \mathcal{E}_{n, j}} \widetilde{P}_{n, j, e_{1}}^{B U, T_{1}}+\sum_{e_{2} \in \mathcal{E}_{n, j}} \widetilde{P}_{n, j, e_{2}}^{B U, T_{2}}\right)+\sum_{e \in \mathcal{E}_{n, j}}\left(\xi^{B} \widetilde{P}_{n, j, e}^{B R, T_{1}}+\xi^{R} \widetilde{P}_{n, j, e}^{R U, T_{2}}\right)\right]}
$$


767 where the auxiliary variable $t$ is given by

$$
t=\frac{1}{P_{T}(\widetilde{\mathcal{P}}, \widetilde{\mathcal{S}})} .
$$

768 Thus, the ESEM problem may be written ${ }^{19}$ as (42)-(53) (see 769 equation at the bottom of the page), where $\widehat{\mathcal{C}}, \widehat{\mathcal{P}}$ and $\widehat{\mathcal{S}}$ indi770 cate the variable-sets containing their associated transformed 771 variables. It is clear that the objective function (42) is affine, 772 hence concave, while the constraints (47)-(53) are all affine, 773 and hence convex. The constraints (43)-(46) are of the form 774 (31) and are hence convex. Therefore, the problem described 775 by (42)-(53) is a concave programming problem, which can be 776 solved using the algorithm of Section V-C.

\footnotetext{
${ }^{19}$ Strictly speaking, the constraint $t>0$ is also needed, but this is guaranteed due to constraint (53).
}

\section{Dual Decomposition Based Solution Algorithm}

777

The dual decomposition method of [17], [41] may be used 778 for conceiving solution algorithms for our SEM and ESEM 779 problems formulated as (20)-(30) and (42)-(53), respectively. 780 We commence by describing the solution algorithm conceived 781 for (42)-(53), which we term the ESEM algorithm. The ESEM 782 algorithm, based on dual decomposition, iterates between cal- 783 culating the tentative optima of the primal variables, namely 784 $\widehat{C}_{n, j, e_{1}}^{B U, T_{1}}, \widehat{C}_{n, j, e_{2}}^{B U, T_{2}}, \widehat{C}_{n, j, e}^{B R U}, \widehat{P}_{n, j, e_{1}}^{B U, T_{1}}, \widehat{P}_{n, j, e_{2}}^{B U, T_{2}}, \widehat{P}_{n, j, e}^{B R, T_{1}}, \widehat{P}_{n, j, e}^{R U, T_{2}}, 785$ $\widehat{s}_{n, j}$ as well as $t$, and updating the dual variables $\lambda^{T_{1}}, \lambda^{T_{2}}, \nu_{m} 786$ as well as $\mu$, which will be defined later, until the objective 787 function value converges.

788

1) Calculating Tentative Optima of Primal Variables: 789 Based on our previous work [17] that employed the dual 790 decomposition and by employing the Karush-Kuhn-Tucker 791

$$
\underset{\widehat{\mathcal{C}}, \widehat{\mathcal{P}}, \widehat{\mathcal{S}}, t}{\operatorname{maximize}} \sum_{i=1}^{N} \sum_{j \in \mathcal{G}_{n}}\left[\sum_{e_{1} \in \mathcal{E}_{n, j}} \widehat{C}_{n, j, e_{1}}^{B U, T_{1}}+\sum_{e_{2} \in \mathcal{E}_{n, j}} \widehat{C}_{n, j, e_{2}}^{B U, T_{2}}\right]+\left[\sum_{e \in \mathcal{E}_{n, j}} \widehat{C}_{n, j, e}^{B R U}\right]
$$

subject to $\frac{\widehat{s}_{n, j}}{2} \log _{2}\left(1+\frac{G_{n, j, e_{1}}^{B U, T_{1}} \widehat{P}_{n, j, e_{1}}^{B U, T_{1}}}{\widehat{s}_{n, j}}\right) \geq \widehat{C}_{n, j, e_{1}}^{B U, T_{1}}, \quad \forall n, j, e_{1}$,

$\frac{\widehat{s}_{n, j}}{2} \log _{2}\left(1+\frac{G_{n, j, e_{2}}^{B U, T_{2}} \widehat{P}_{n, j, e_{2}}^{B U, T_{2}}}{\widehat{s}_{n, j}}\right) \geq \widehat{C}_{n, j, e_{2}}^{B U, T_{2}}, \quad \forall n, j, e_{2}$,

$\frac{\widehat{s}_{n, j}}{2} \log _{2}\left(1+\frac{G_{n, j, e}^{B R, T_{1}} \widehat{P}_{n, j, e}^{B R, T_{1}}}{\widehat{s}_{n, j}}\right) \geq \widehat{C}_{n, j, e}^{B R U}, \quad \forall n, j, e$,

$\frac{\widehat{s}_{n, j}}{2} \log _{2}\left(1+\frac{G_{n, j, e}^{R U, T_{2}} \widehat{P}_{n, j, e}^{R U, T_{2}}}{\widehat{s}_{n, j}}\right) \geq \widehat{C}_{n, j, e}^{B R U}, \quad \forall n, j, e$,

$\sum_{j \in \mathcal{G}_{n}} \widehat{s}_{n, j} \leq t, \quad \forall n$,

$\sum_{i=1}^{N} \sum_{j \in \mathcal{G}_{n}}\left[\sum_{e_{1} \in \mathcal{E}_{n, j}} \widehat{P}_{n, j, e_{1}}^{B U, T_{1}}+\sum_{e \in \mathcal{E}_{n, j}} \widehat{P}_{n, j, e}^{B R, T_{1}}\right] \leq t \cdot P_{\text {max }}^{B}$,

$\sum_{i=1}^{N} \sum_{j \in \mathcal{G}_{n}} \sum_{e_{2} \in \mathcal{E}_{n, j}} \widehat{P}_{n, j, e_{2}}^{B U, T_{2}} \leq t \cdot P_{\max }^{B}$

$\sum_{i=1}^{N} \sum_{j \in \mathcal{G}_{n}} \sum_{\substack{e \in \mathcal{E}_{n, j} \\ \mathcal{M}(e)=m}} \widehat{P}_{n, j, e}^{R U, T_{2}} \leq t \cdot P_{\max }^{R}, \quad \forall m$,

$0 \leq \widehat{s}_{n, j} \leq t, \quad \forall n, j$,

$\widehat{P}_{n, j, e_{1}}^{B U, T_{1}}, \widehat{P}_{n, j, e}^{B R, T_{1}}, \widehat{P}_{n, j, e_{2}}^{B U, T_{2}}, \widehat{P}_{n, j, e}^{R U, T_{2}} \geq 0, \quad \forall n, j, e_{1}, e_{2}, e$,

$t \cdot\left(P_{C}^{B}+M \cdot P_{C}^{R}\right)$

$$
+\frac{1}{2} \sum_{n=1}^{N} \sum_{j \in \mathcal{G}_{n}}\left[\xi^{B}\left(\sum_{e_{1} \in \mathcal{E}_{n, j}} \widehat{P}_{n, j, e_{1}}^{B U, T_{1}}+\sum_{e_{2} \in \mathcal{E}_{n, j}} \widehat{P}_{n, j, e_{2}}^{B U, T_{2}}\right)+\sum_{e \in \mathcal{E}_{n, j}}\left(\xi^{B} \widehat{P}_{n, j, e}^{B R, T_{1}}+\xi^{R} \widehat{P}_{n, j, e}^{R U, T_{2}}\right)\right]=1
$$


792 optimality conditions [32], we reveal that the tentatively opti793 mal transformed power control variables for the direct SMCs 794 encountered in the problem of (42)-(53) may be formulated as 795 the water-filling solutions of ${ }^{20}$

$$
\begin{aligned}
\widehat{P}_{n, j, e_{1}}^{B U, T_{1}} & =\widehat{s}_{n, j}\left[\frac{1}{\left(\xi^{B} \mu+2 \lambda^{T_{1}}\right) \ln 2}-\frac{1}{G_{n, j, e_{1}}^{B U, T_{1}}}\right]^{+} \\
& =\widehat{s}_{n, j} P_{n, j, e_{1}}^{B U, T_{1}}
\end{aligned}
$$

796 and

$$
\begin{aligned}
\widehat{P}_{n, j, e_{2}}^{B U, T_{2}} & =\widehat{s}_{n, j}\left[\frac{1}{\left(\xi^{B} \mu+2 \lambda^{T_{2}}\right) \ln 2}-\frac{1}{G_{n, j, e_{2}}^{B U, T_{2}}}\right]^{+} \\
& =\widehat{s}_{n, j} P_{n, j, e_{2}}^{B U, T_{2}} .
\end{aligned}
$$

797 Furthermore, the transformed power control variables for the 798 relaying SMCs may be initially written as

$$
\begin{aligned}
\widehat{P}_{n, j, e}^{B R, T_{1}} & =\widehat{s}_{n, j}\left[\frac{1}{\left(\xi^{B} \mu+2 \lambda^{T_{1}}\right) \ln 2}-\frac{1}{G_{n, j, e}^{B R, T_{1}}}\right]^{+} \\
& =\widehat{s}_{n, j} P_{n, j, e}^{B R, T_{1}}
\end{aligned}
$$

799 and

$$
\begin{aligned}
\widehat{P}_{n, j, e}^{R U, T_{2}} & =\widehat{s}_{n, j}\left[\frac{1}{\left(\xi^{R} \mu+2 \nu_{\mathcal{M}(e)}\right) \ln 2}-\frac{1}{G_{n, j, e}^{R U, T_{2}}}\right]^{+} \\
& =\widehat{s}_{n, j} P_{n, j, e}^{R U, T_{2}} .
\end{aligned}
$$

800 Note that the value of $\widehat{s}_{n, j}$ in (54)-(57) is not yet known. Since 801 the SE attainable for a relaying link is limited by the weaker of 802 the BS-to-RN and RN-to-UE links, there is no need to transmit 803 at a high power on the stronger link, if the other link is unable 804 to support the high SE. Thus, the tentatively optimal trans805 formed power control variables provided for the relaying SMC $806 e$ may be refined by substituting (56), (57) into the right-hand 807 side of

$$
\widehat{P}_{n, j, e}^{B R, T_{1}}=\min \left(\widehat{P}_{n, j, e}^{B R, T_{1}}, \frac{G_{n, j, e}^{R U, T_{2}}}{G_{n, j, e}^{B R, T_{1}}} \cdot \widehat{P}_{n, j, e}^{R U, T_{2}}\right)
$$

808 and

$$
\widehat{P}_{n, j, e}^{R U, T_{2}}=\min \left(\widehat{P}_{n, j, e}^{R U, T_{2}}, \frac{G_{n, j, e}^{B R, T_{1}}}{G_{n, j, e}^{R U, T_{2}}} \cdot \widehat{P}_{n, j, e}^{B R, T_{1}}\right) .
$$

\footnotetext{
${ }^{20}$ In this paper, $[\cdot]^{+}$is equivalent to $\max (0, \cdot)$.
}

As a result, the tentative estimates of the maximum values 809 that $\widehat{C}_{n, j, e_{1}}^{B U, T_{1}}, \widehat{C}_{n, j, e_{2}}^{B U, T_{2}}$ and $\widehat{C}_{n, j, e}^{B R U}$ can attain are given by

$$
\begin{aligned}
& \widehat{C}_{n, j, e_{1}}^{B U, T_{1}}=\frac{\widehat{s}_{n, j}}{2} \log _{2}\left(1+\frac{G_{n, j, e_{1}}^{B U, T_{1}} \widehat{P}_{n, j, e_{1}}^{B U, T_{1}}}{\widehat{s}_{n, j}}\right), \\
& \widehat{C}_{n, j, e_{2}}^{B U, T_{2}}=\frac{\widehat{s}_{n, j}}{2} \log _{2}\left(1+\frac{G_{n, j, e_{2}}^{B U, T_{2}} \widehat{P}_{n, j, e_{2}}^{B U, T_{2}}}{\widehat{s}_{n, j}}\right),
\end{aligned}
$$

and

$$
\begin{aligned}
\widehat{C}_{n, j, e}^{B R U} & =\frac{\widehat{s}_{n, j}}{2} \log _{2}\left(1+\frac{G_{n, j, e}^{B R, T_{1}} \widehat{P}_{n, j, e}^{B R, T_{1}}}{\widehat{s}_{n, j}}\right) \\
& =\frac{\widehat{s}_{n, j}}{2} \log _{2}\left(1+\frac{G_{n, j, e}^{R U, T_{2}} \widehat{P}_{n, j, e}^{R U, T_{2}}}{\widehat{s}_{n, j}}\right),
\end{aligned}
$$

where the value of $\widehat{s}_{n, j}$ remains unknown. However, it is plau- 812 sible that for the purpose of maximizing the objective function 813 value, $\widehat{s}_{n, j}, \forall n, j$ will always be given its maximum value $t$, if 814 the single SMC group $j$ is selected for subcarrier block $n$. Thus, 815 the tentatively optimal SMC group $j$ for subcarrier block $n$ is 816 given by the group obtaining the highest value of

$$
\sum_{j \in \mathcal{G}_{n}}\left[\sum_{e_{1} \in \mathcal{E}_{n, j}} \widehat{C}_{n, j, e_{1}}^{B U, T_{1}}+\sum_{e_{2} \in \mathcal{E}_{n, j}} \widehat{C}_{n, j, e_{2}}^{B U, T_{2}}\right]+\sum_{e \in \mathcal{E}_{n, j}} \widehat{C}_{n, j, e}^{B R U} .
$$

where $\widehat{s}_{n, j}$ inside the logarithm functions may be canceled 818 out. Additionally, we can ignore the common positive 819 multiplicative factor of $t$ without affecting the maximization of 820 (63). The objective function (42) is maximized when choosing 821 this particular group $j$ for subcarrier block $n$, while for the 822 remaining groups associated with the same subcarrier block, we 823 set $\widehat{P}_{n, j^{\prime} \neq j, e_{1}}^{B U, T_{1}}=\widehat{P}_{n, j^{\prime} \neq j, e_{2}}^{B U, T_{2}}=\widehat{P}_{n, j^{\prime} \neq j, e}^{B R, T_{1}}=\widehat{P}_{n, j^{\prime} \neq j, e}^{R U, T_{2}}=\widehat{s}_{n, j^{\prime} \neq j}=824$ $\widehat{C}_{n, j^{\prime} \neq j, e_{1}}^{B U, T_{1}}=\widehat{C}_{n, j^{\prime} \neq j, e_{2}}^{B U, T_{2}}=\widehat{C}_{n, j^{\prime} \neq j, e}^{B R U}=P_{n, j^{\prime} \neq j, e_{1}}^{B U, T_{1}}=P_{n, j^{\prime} \neq j, e_{2}}^{B U, T_{2}}=825$ $P_{n, j^{\prime} \neq j, e}^{B R, T_{1}}=P_{n, j^{\prime} \neq j, e}^{R U, T_{2}}=0$, as these remaining groups are not 826 chosen.

Consequently, the value of $t$ is given by (64) (see equation 828 at the bottom of the page). Note that this is possible without 829 knowing the exact value of $\widehat{s}_{n, j}$, since the factor of $\widehat{s}_{n, j}$ may 830 be canceled out, and thus (64) is only dependent on the dual 831 variables and on the tentatively optimal SMC group selection. 832

Having identified the tentative optimal SMC group, we set 833 $\widehat{s}_{n, j}=t$ for this selected SMC group corresponding to each 834 subcarrier block $n$, and we have

$$
\begin{aligned}
\widehat{C}_{n, j, e_{1}}^{B U, T_{1}} & =\frac{t}{2} \log _{2}\left(1+G_{n, j, e_{1}}^{B U, T_{1}} P_{n, j, e_{1}}^{B U, T_{1}}\right), \\
\widehat{C}_{n, j, e_{2}}^{B U, T_{2}} & =\frac{t}{2} \log _{2}\left(1+G_{n, j, e_{2}}^{B U, T_{2}} P_{n, j, e_{2}}^{B U, T_{2}}\right)
\end{aligned}
$$

$$
t=\left(P_{C}^{B}+M \cdot P_{C}^{R}+\frac{1}{2} \sum_{n=1}^{N} \sum_{j \in \mathcal{G}_{n}}\left[\xi^{B}\left(\sum_{e_{1} \in \mathcal{E}_{n, j}} P_{n, j, e_{1}}^{B U, T_{1}}+\sum_{e_{2} \in \mathcal{E}_{n, j}} P_{n, j, e_{2}}^{B U, T_{2}}\right)+\sum_{e \in \mathcal{E}_{n, j}}\left(\xi^{B} P_{n, j, e}^{B R, T_{1}}+\xi^{R} P_{n, j, e}^{R U, T_{2}}\right)\right]\right)^{-1}
$$


836 as well as

$$
\begin{aligned}
\widehat{C}_{n, j, e}^{B R U} & =\frac{t}{2} \log _{2}\left(1+G_{n, j, e}^{B R, T_{1}} P_{n, j, e}^{B R, T_{1}}\right) \\
& =\frac{t}{2} \log _{2}\left(1+G_{n, j, e}^{R U, T_{2}} P_{n, j, e}^{R U, T_{2}}\right),
\end{aligned}
$$

837 for that selected SMC group. To summarize, given a set of dual 838 variables, the values of power control variables are obtained, 839 resulting in an tentatively optimal SMC group, which obtains 840 the SE values for the corresponding subcarrier block. There841 fore, all of the primal variables are obtained for a given set of 842 dual variables. Thus, they are jointly optimized.

843 2) Updating the Dual Variables: From the derivation of the 844 optimal primal variables described in Section V-C.1, we can 845 see that the constraints (43)-(47) and (51)-(53) are implicitly 846 satisfied. Therefore, we update the dual variables $\lambda^{T_{1}}, \lambda^{T_{2}}$ 847 and $\nu_{m}$ which are associated with the remaining constraints 848 (48)-(50), respectively. These may be viewed as pricing param849 eters to ensure that the optimal power control variables satisfy 850 (48)-(50).

851 Since the Lagrangian of (42)-(53) is differentiable w.r.t. the 852 dual variables, at each iteration $i$ of the solution algorithm, 853 these dual variables may be updated according to (68)-(70) (see equation at the bottom of the page), where $\delta_{\lambda^{T_{1}}}(i), \delta_{\lambda^{T_{2}}}(i) 854$ and $\delta_{\nu_{m}}(i)$ are appropriately chosen step sizes [41] at iteration $i .855$

The remaining dual variable, $\mu$, which is associated with 856 (53) must also be updated. However, the constraint given by 857 (53) is implicitly satisfied since the value of $t$ is computed 858 from (64). Therefore, we opt for an alternative method based 859 on differentiating the Lagrangian w.r.t $t$ and substituting in the 860 intermediate values of $\widehat{\mathcal{C}}, \widehat{\mathcal{P}}, \widehat{\mathcal{S}}$ and $t$. Thus, the updated value 861 of $\mu$ is given by (71).

862

All primal variables are jointly optimized in Section V-C.1 as 863 the optimal power variables are determined by the related dual 864 variables. This leads to the optimal group selection and rate vari- 865 ables, which then allow us to find the optimal $t$. Given the tenta- 866 tive optima of primal variables, the algorithm proceeds to update 867 the dual variables, which are mostly to ensure that the maximum 868 power constraints are not violated. Using these updated dual 869 variables, the algorithm repeats this process until the objective 870 function value $\widehat{\eta}_{E}(i)$ at iteration $i$ reaches the predefined con- 871 vergence threshold, which is given by $\left|\widehat{\eta}_{E}(i)-\widehat{\eta}_{E}(i-1)\right|<\epsilon . \quad 872$

The method presented in Section V-C.1 and Section V-C.2 873 solves the ESEM problem described by (42)-(53). It may also 874 be invoked for solving the SEM problem of (20)-(30), while 875 fixing $\mu=0$ and $t=1$. This is because the ESEM problem 876 considered is simplified to the SEM problem, when we have 877 $\mu=0$ and $t=1$.

$$
\begin{aligned}
& \lambda^{T_{1}}(i)=\left[\lambda^{T_{1}}(i-1)-\delta_{\lambda^{T_{1}}}(i) \cdot\left(t \cdot P_{\max }^{B}-\sum_{i=1}^{N} \sum_{j \in \mathcal{G}_{n}}\left[\sum_{e_{1} \in \mathcal{E}_{n, j}} \widehat{P}_{n, j, e_{1}}^{B U, T_{1}}+\sum_{e \in \mathcal{E}_{n, j}} \widehat{P}_{n, j, e}^{B R, T_{1}}\right]\right]^{+}\right. \\
& \lambda^{T_{2}}(i)=\left[\lambda^{T_{2}}(i-1)-\delta_{\lambda^{T_{2}}}(i) \cdot\left(t \cdot P_{\max }^{B}-\sum_{i=1}^{N} \sum_{j \in \mathcal{G}_{n}} \sum_{e_{2} \in \mathcal{E}_{n, j}} \widehat{P}_{n, j, e_{2}}^{B U, T_{2}}\right)\right]^{4}
\end{aligned}
$$

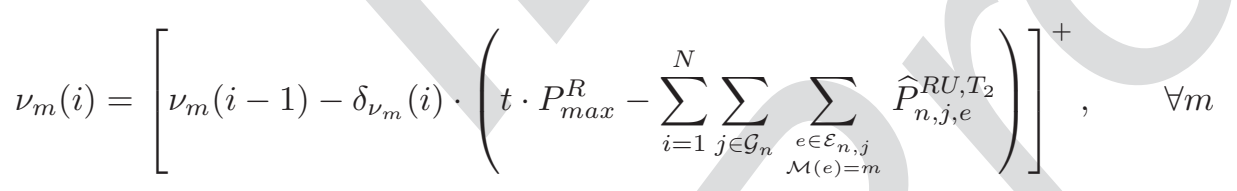

$$
\begin{aligned}
& \mu(i)=t \cdot\left(\sum_{i=1}^{N} \sum_{j \in \mathcal{G}_{n}}\left[\sum_{e_{1} \in \mathcal{E}_{n, j}} \widetilde{C}_{n, j, e_{1}}^{B U, T_{1}}+\sum_{e_{2} \in \mathcal{E}_{n, j}} \widetilde{C}_{n, j, e_{2}}^{B U, T_{2}}\right]+\left[\sum_{e \in \mathcal{E}_{n, j}} \widetilde{C}_{n, j, e}^{B R U}\right]\right. \\
& +\lambda^{T_{1}}(i-1) \cdot\left(P_{\max }^{B}-\sum_{i=1}^{N} \sum_{j \in \mathcal{G}_{n}}\left[\sum_{e_{1} \in \mathcal{E}_{n, j}} \widetilde{P}_{n, j, e_{1}}^{B U, T_{1}}+\sum_{e \in \mathcal{E}_{n, j}} \widetilde{P}_{n, j, e}^{B R, T_{1}}\right]\right) \\
& +\lambda^{T_{2}}(i-1) \cdot\left(P_{\max }^{B}-\sum_{i=1}^{N} \sum_{j \in \mathcal{G}_{n}} \sum_{e_{2} \in \mathcal{E}_{n, j}} \widetilde{P}_{n, j, e_{2}}^{B U, T_{2}}\right) \\
& \left.+\sum_{m=1}^{M} \nu_{m}(i-1) \cdot\left(P_{\max }^{R}-\sum_{i=1}^{N} \sum_{j \in \mathcal{G}_{n}} \sum_{\substack{e \in \mathcal{E}_{n, j} \\
\mathcal{M}(e)=m}} \widetilde{P}_{n, j, e}^{R U, T_{2}}\right)\right)
\end{aligned}
$$


TABLE I

Simulation Parameters Used to Obtain All Results IN SECTION VI UNLESS OTHERWISE SPECIFIED

\begin{tabular}{|l|r|}
\hline Simulation parameter & Value \\
\hline \hline Subcarrier block bandwidth, $W$ [Hertz] & $180 \mathrm{k}$ \\
\hline Number of RNs, $M$ & $\{0,1,2,4\}$ \\
\hline Number of subcarriers blocks, $N$ & $\{2,10\}$ \\
\hline Number of UEs, $K$ & $(4,4,2)$ \\
\hline Antenna configuration, $\left(N_{B}, N_{R}, N_{U}\right)$ & 0.5 \\
\hline Cell radius, [km] & $\{0.75,1.25,1.75,2.25\}$ \\
\hline $\begin{array}{l}\text { Ratio of BS-to-RN distance to the cell } \\
\text { radius, } D_{r}\end{array}$ & 0 \\
\hline SNR gap of wireless transceivers, $\Delta \gamma$ [dB] & \\
\hline $\begin{array}{l}\text { Maximum total transmission power of the } \\
\text { BS and RNs, } P_{\text {max }}^{B} \text { and } P_{\text {max }}^{R}[\mathrm{dBm}]\end{array}$ & $30,20,30,60\}$ \\
\hline $\begin{array}{l}\text { Fixed power rating of the BS, } \\
P_{C}^{(B)} \text { [Watts] [36], [42] }\end{array}$ & $32.306 N_{B}$ \\
\hline $\begin{array}{l}\text { Fixed power rating of RNs, } \\
P_{C}^{(R)} \text { [Watts] [36], [42] }\end{array}$ & $21.874 N_{R}$ \\
\hline $\begin{array}{l}\text { Reciprocal of the BS power amplifier's } \\
\text { drain efficiency, } \xi^{(B)}[36],[42]\end{array}$ & $3.24 N_{B}$ \\
\hline $\begin{array}{l}\text { Reciprocal of the RNs' power amplifier's } \\
\text { drain efficiency, } \xi^{(R)}[36],[42]\end{array}$ & $4.04 N_{R}$ \\
\hline Noise power spectral density, $N_{0}$ [dBm/Hz] & -174 \\
\hline Convergence threshold, $\epsilon$ & $10^{-6}$ \\
\hline Number of channel samples & $10^{4}$ \\
\hline
\end{tabular}

\section{NUMERICAL RESULTS AND DisCUSSIONS}

880 This section presents the numerical results obtained, when 881 employing the SEM and ESEM algorithms ${ }^{21}$ described in 882 Section V to the MIMO-OFDMA multi-relay cellular network 883 considered. The pertinent simulation parameters are given 884 in Table I. Additionally, the path-loss effect is characterized 885 relying on the method and parameters of [30], where the BS886 to-UE and RN-to-UE links are assumed to be non-line-of-sight 887 (NLOS) links, since these links are typically blocked by 888 buildings and other large obstructing objects, while the BS-to$889 \mathrm{RN}$ links are realistically assumed to be line-of-sight (LOS) 890 links, as the RNs may be strategically deployed on tall buildings 891 to create strong wireless backhaul links. Furthermore, indepen892 dently and randomly generated set of UE locations as well as 893 fading channel realizations were used for each channel sample. 894 The results of a baseline algorithm is also presented to high895 light the improved performance obtained from employing the 896 SEM and ESEM algorithms. This baseline algorithm consists 897 of a random SMC grouping (RG) selection for each subcarrier 898 block and then equal power allocation (EPA) across all the 899 selected SMCs, and will be termed the RG-EPA algorithm.

\section{A. On the Optimality and the Relative Complexity of ESGA 901 and OCGA for Various $\alpha$ Values}

902 Firstly, the behavior of the ESGA and OCGA as a function 903 of $\alpha$ is examined. Note in Fig. 4 that since the ESGA is

\footnotetext{
${ }^{21}$ In all cases, the step sizes and the initial values of the dual variables described in Section V-C.2 are empirically optimized to give the optimal objective function value in as few iterations as possible, although the exact analytical method for determining the optimal step sizes and initial values still remains an open issue. In our experience, the algorithms converge within just 10 iterations when carefully chosen step sizes are employed, regardless of the size of the problem.
}

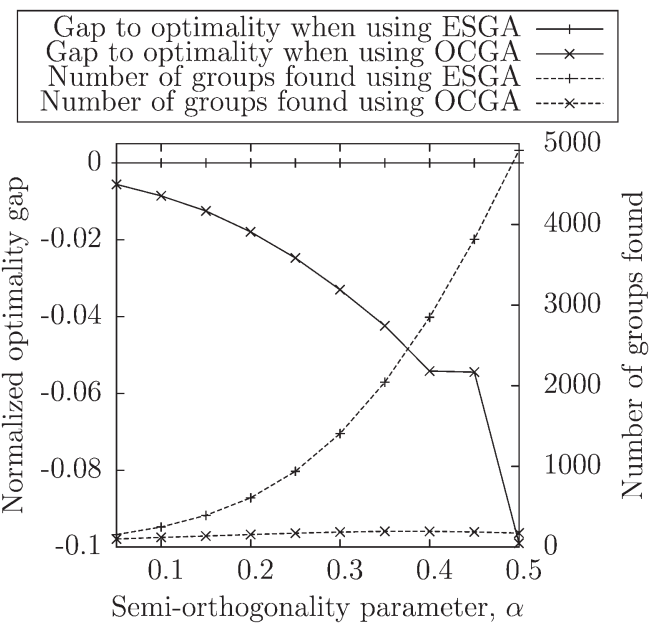

Fig. 4. The optimality gap and total number of SMC groups found when employing the ESGA and OCGA, and using the parameters in Table I with $N=6, K=2, M=2, P_{\max }^{B}=20 \mathrm{dBm}, P_{\max }^{R}=10 \mathrm{dBm}$ and a cell radius of $0.75 \mathrm{~km}$.

capable of enumerating all possible SMC groupings, which 904 satisfy (1) for the corresponding $\alpha$, the optimal SE is attained. 905 The 'normalized optimality gap' is then defined as $\left(\beta / \beta^{*}\right)-1,906$ where $\beta^{*}$ is the optimal SE obtained from employing the ESGA 907 algorithm, and $\beta$ is the SE obtained from any other algorithm. 908 We can see from Fig. 4, that the normalized optimality gap 909 of OCGA relative to ESGA is about $-0.005 \sim-0.1$ for the 910 $\alpha$ values considered. However, the number of groups found 911 using ESGA is exponentially increasing with $\alpha$. By contrast, for 912 OCGA, this number is always significantly lower and gradually 913 becomes less than 200, when $\alpha$ increases to 0.5 . In fact, the 914 number of groups found by OCGA is reduced to about 3.5\% 915 of that found by ESGA at $\alpha=0.5$. This demonstrates the 916 viability of using OCGA in the following simulations as a 917 reduced-complexity near-optimum alternative to ESGA. Under 918 the conditions considered in Fig. 4, the optimal ESE solution 919 is the same as the optimal SE solution, as detailed in the next 920 subsection. Therefore, as far as ESEM is concerned, similar 921 conclusions may be drawn regarding the optimality of the two 922 grouping algorithms.

B. The Variation in Achievable SE and ESE for Different Values of $P_{\max }^{B}$ and $P_{\max }^{R}$

As shown in Fig. 5(a), the achievable SE is monotonically in- 926 creasing with $P_{\max }^{B}$ and $P_{\max }^{R}$ when using the SEM algorithm. 927 This is not unexpected, since the SEM algorithm optimally 928 allocates all the available power for the sake of achieving the 929 maximum SE. By comparison, we observed from Fig. 5(a) 930 and (b) that both the achievable SE and ESE of the ESEM 931 algorithm saturate at some moderate values of $P_{\max }^{B}$ and/or 932 $P_{\max }^{R}$. This is because the ESEM algorithm only allocates just 933 enough power (that may be lower than the power budget values 934 of $P_{\max }^{B}$ and/or $P_{\max }^{R}$ ) for the sake of achieving the maximum 935 ESE. On the other hand, the ESE performance of the SEM algo- 936 rithm is severely degraded upon further increasing $P_{\max }^{B}$ and/or 937 $P_{\max }^{R}$ after its ESE performance reaches the peak, as shown in 938 Fig. 5(b). This is because the ESE metric is a quasiconcave 939 


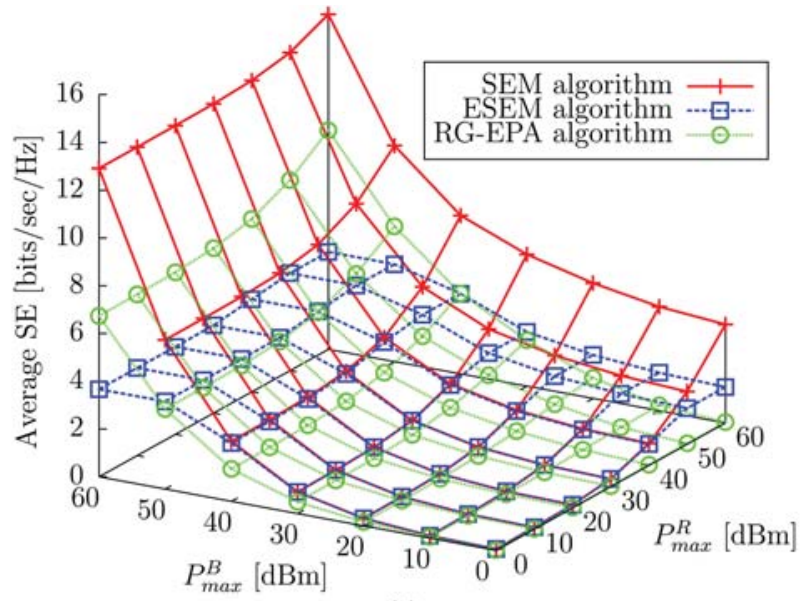

(a)

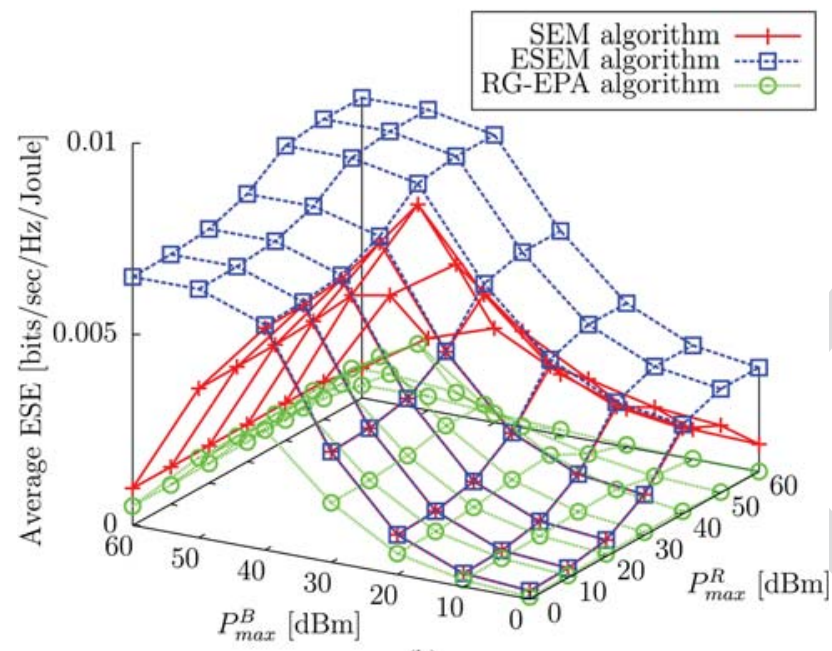

(b)

Fig. 5. The average achievable SE and ESE of the SEM, ESEM and RGEPA algorithms upon varying $P_{\max }^{B}$ and $P_{\max }^{R}$. The parameters in Table I with $N=6, K=10, M=2, \alpha=0.1$ and a cell radius of $1.75 \mathrm{~km}$ are used. (a) Surface plots of the achievable SE when using the SEM, ESEM and RGEPA algorithms. (b) Surface plots of the achievable ESE when using the SEM, ESEM and RG-EPA algorithms.

940 function of the transmit powers-its numerator (i.e. the SE) 941 increases logarithmically with the transmit powers, while its 942 denominator increases linearly with the transmit powers. In 943 fact, the peak ESE of the SEM algorithm is attained at $P_{\max }^{B}=$ $94440 \mathrm{dBm}$ and $P_{\max }^{R}=40 \mathrm{dBm}$, as seen in Fig. 5(b), and the 945 associated normalized optimality gap is only -0.074 . By con946 trast, the achievable ESE when using the ESEM algorithm also 947 saturates at around $P_{\max }^{B}=40 \mathrm{dBm}$ and $P_{\max }^{R}=40 \mathrm{dBm} .^{22}$ 948 Thus, the operating point of " $P_{\max }^{B}=40 \mathrm{dBm}$ and $P_{\max }^{R}=$ $94940 \mathrm{dBm}$ " may strike an attractive balance between SEM and 950 ESEM. Of course, the required trade-off may be struck on a 951 case-by-case basis in practical systems.

952 Additionally, the RG-EPA algorithm performs significantly 953 worse in terms of SE when compared to the SEM algorithm, 954 and in terms of ESE when compared to the ESEM algorithm. 955 Furthermore, the RG-EPA algorithm performs even worse than 956 the SEM algorithm in terms of ESE. Although the obtained SE

${ }^{22}$ Note that when $P_{\max }^{B}$ and $P_{\max }^{R}$ have low/moderate values, the SEM and ESEM algorithms share the same solutions of $\mathcal{P}$ and $\mathcal{S}$.

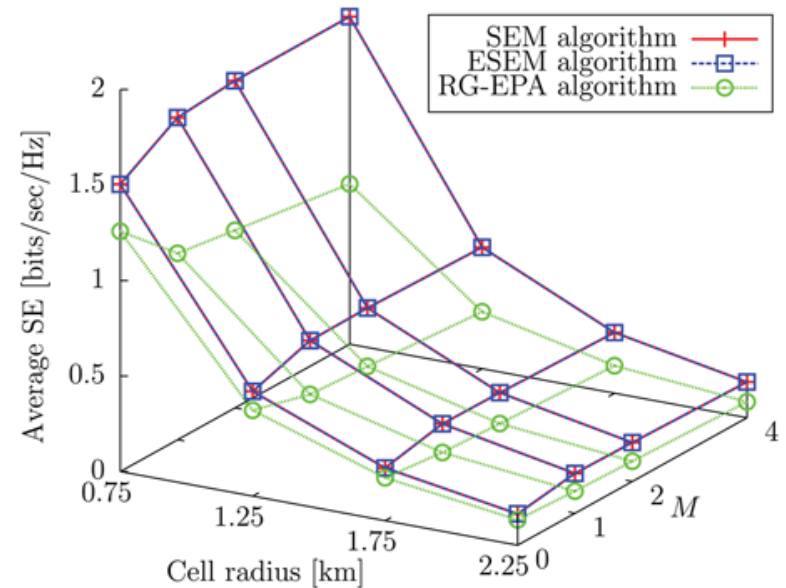

(a)

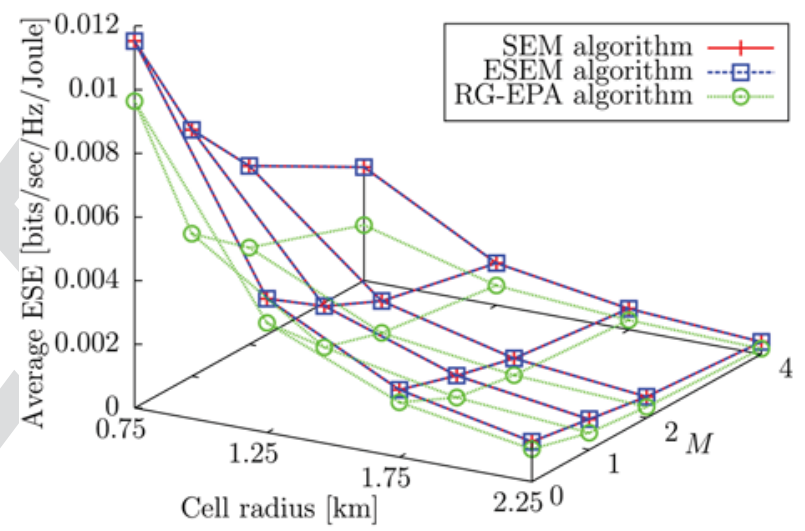

(b)

Fig. 6. The average achievable SE and ESE of the SEM, ESEM and RG-EPA algorithms upon varying $M$ and cell radius, and using the parameters in Table I with $N=6, K=10, \alpha=0.1, P_{\max }^{B}=20 \mathrm{dBm}$ and $P_{\max }^{R}=10 \mathrm{dBm}$. (a) Surface plots of the achievable SE when using the SEM, ESEM and RGEPA algorithms. (b) Surface plots of the achievable ESE when using the SEM, ESEM and RG-EPA algorithms.

when using the RG-EPA algorithm is, in some cases, higher 957 than the SE obtained when using the ESE algorithm, this 958 performance improvement comes at a great cost to the ESE 959 performance of the RG-EPA algorithm.

960

Finally, note that although both the SE of the SEM algorithm, 961 and the ESE of the ESEM algorithm are non-decreasing as 962 either $P_{\max }^{B}$ or $P_{\max }^{R}$ is increased, the effect of increasing $P_{\max }^{B} 963$ on the SE or ESE is significantly more pronounced, than that of 964 applying the same increase to $P_{\max }^{R}$. The intuitive reasoning 965 behind this is that the power available at the BS has a more 966 pronounced effect on the system's performance, since the direct 967 links and, more importantly, the BS-to-RN links rely on the BS. 968 Therefore, increasing $P_{\max }^{R}$ is futile if the BS-to-RN links are 969 not allocated sufficient power to support the RN-to-UE links. 970

\section{The Achievable SE and ESE as a Function of $M$ and the Cell Radius}

Fig. 6 illustrates some advantages and disadvantages of em- 973 ploying RNs in the cellular system considered. We observe that 974 the specific low values of the power constraints result in the 975 same solutions for both the SEM and ESEM algorithms. This 976 phenomenon was also shown in Fig. 5. 
978 As evidenced in Fig. 6(a), the attainable SE increases with $979 \mathrm{M}$, which is a benefit of the additional selection diversity, when 980 forming relaying links. However, the attainable SE does not 981 increase substantially beyond $M=2$. In fact, only an increase 982 of $0.1 \%$ is attained for the SE when $M$ is increased from 2 to 9834 at a cell radius of $0.75 \mathrm{~km}$. On the other hand, the cost in 984 terms of ESE is significant (36.4\%), as shown in Fig. 6(b). This 985 suggests that employing RNs does not constitute an energy986 spectral-efficient technique although it increases the SE of a 987 cellular system, which is partially due to the power amplifier 988 inefficiency and owing to the non-negligible fixed circuit energy 989 dissipation. Note furthermore that both the attainable SE and 990 ESE are decreasing upon increasing the cell radius as a result of 991 the increased path-loss of all the wireless links. However, this 992 reduction is relatively small between a cell radius of $1.75 \mathrm{~km}$ 993 and $2.25 \mathrm{~km}$. The reason behind this phenomenon is that both 994 the SEM and ESEM algorithms will selectively serve the UEs 995 nearer to the BS, so that a similar performance may be attained 996 without suffering from a substantial path-loss. This is also the 997 reason why the gain in SE gleaned by employing RNs at a cell 998 radius of $2.25 \mathrm{~km}$ seems negligible in Fig. 6(a). Once again, 999 the RG-EPA algorithm performs worse both in terms of SE and 1000 ESE performance.

\section{D. The Achievable SE and ESE as a Function of $N$ and $N_{B}$}

1002 Fig. 7 illustrates the effect of increasing $N$ and $N_{B}$ on the 1003 attainable SE and ESE. Note that in a similar fashion to Fig. 6, 1004 the SEM and ESEM algorithms attain the same solutions in the 1005 operating region considered.

1006 Observe from both Fig. 7(a) and (b) that the attainable SE and 1007 ESE increase upon increasing $N_{B}$. This is due to the increased 1008 attainable spatial degrees of freedom at the BS in the first 1009 transmission phase, which allows for more direct transmissions 1010 overall. However, both the SE and ESE are reduced upon 1011 increasing $N$, which suggests that increasing the number of 1012 subcarrier blocks does not increase the average efficiency of 1013 each block. This is because the power constraints are fixed 1014 and thus there is insufficient power for fully exploiting the 1015 additional subcarrier blocks. However, note that both total SE 1016 and ESE do indeed increase upon increasing $N$, which may be 1017 explicitly seen upon multiplying the results of Fig. 7(a) and (b) 1018 by $N W$. The RG-EPA algorithm performs worse in both cases 1019 as expected.

\section{CONCLUSION AND FUturE WORK}

1021 In this paper, firstly a novel transmission protocol based on 1022 joint transmit-BF and receive-BF was developed for the multi1023 relay MIMO-OFDMA cellular network considered. This proto$1024 \mathrm{col}$ allows for achieving high-SE performance for the MIMO 1025 broadcast network consisting of a BS, multiple RNs and multi1026 ple UEs. The associated MIMO channel matrices were mathe1027 matically decomposed into multiple MISO channels, which we 1028 referred to as SMCs, using receive-BF. By applying ZFBF at the 1029 transmitter, the interference between SMC-based concurrent 1030 transmissions is completely eliminated, provided that perfect 1031 CSI-knowledge is available. For the purposes of obtaining a

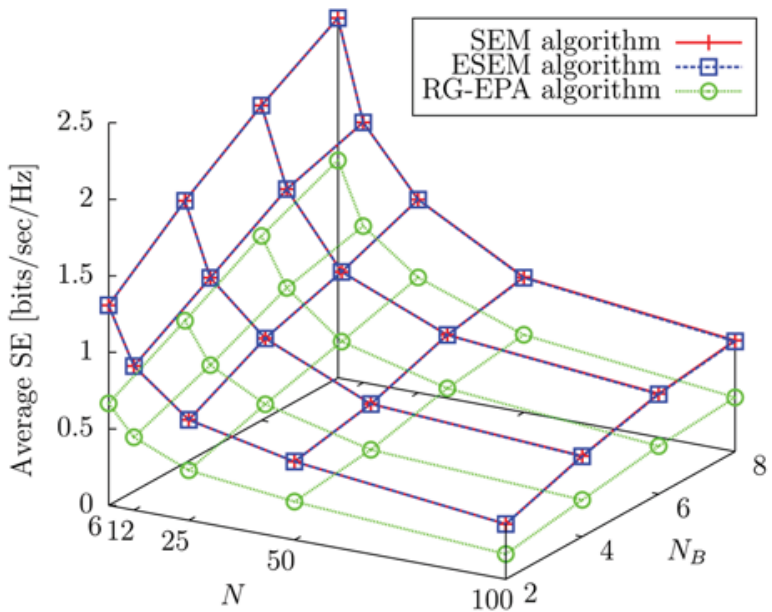

(a)

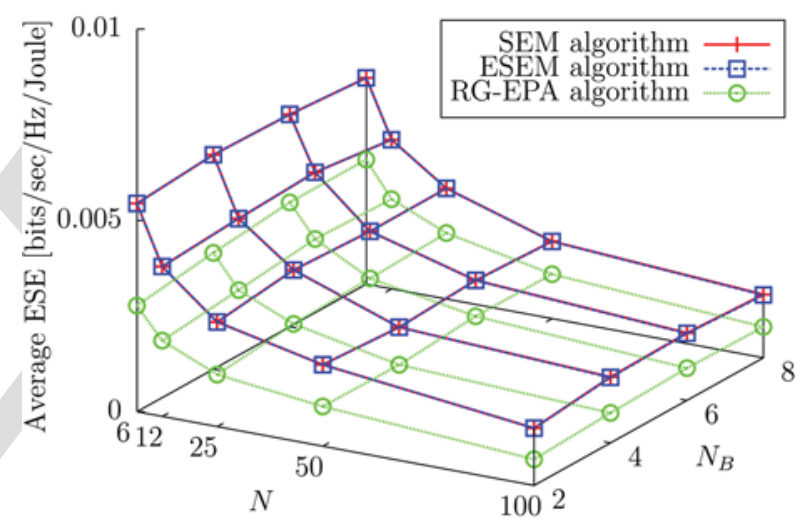

(b)

Fig. 7. The average achievable SE and ESE of the SEM, ESEM and RG-EPA algorithms upon varying $N$ and $N_{B}$, and using the parameters in Table I with $M=2, K=10, \alpha=0.1, P_{\max }^{B}=20 \mathrm{dBm}, P_{\max }^{R}=10 \mathrm{dBm}$ and a cell radius of $0.75 \mathrm{~km}$. (a) Surface plots of the achievable SE when using the SEM, ESEM and RG-EPA algorithms. (b) Surface plots of the achievable ESE when using the SEM, ESEM and RG-EPA algorithms.

higher multiplexing gain, the SMCs may be grouped according 1032 to the semi-orthogonality criterion. Consequently, a pair of 1033 grouping algorithms were proposed, referred to as ESGA and 1034 OCGA. The former exhaustively enumerates all of the possible 1035 groupings, whereas the latter aims to be a lower-complexity de- 1036 sign alternative. Finding the SE-optimal and ESE-optimal SMC 1037 groupings as well as their associated optimal power control 1038 variables were formulated as optimization problems. With the 1039 aid of several variable relaxations and transformations, these 1040 optimization problems were transformed into concave opti- 1041 mization problems. Thus, the dual decomposition approach was 1042 employed for finding the optimal solutions. We demonstrated 1043 that the OCGA constitutes an attractive alternative to ESGA, 1044 since it offers a near-optimal performance at a substantially re- 1045 duced complexity. Furthermore, several numerical results were 1046 presented for characterizing the system's attainable SE and ESE 1047 performance across a wide range of system parameters, such 1048 as the transmit power constraints, cell radius, the number of 1049 RNs, the number of BS antennas and the number of subcarrier 1050 blocks. Additionally, we demonstrated that our SEM/ESEM 1051 algorithms perform significantly better than the benchmark RG- 1052 EPA algorithm. 
1054 In our future work, we will consider unity frequency reuse 1055 multi-relay multi-cell networks. Thus, these networks are 1056 interference-limited, rather than noise-limited. Consequently, 1057 improved transmission protocols and optimization methods are 1058 required for managing both the intra-cell and inter-cell interfer1059 ence to improve the system's SE and ESE performance.

1060

1061

1062

1063

1064

1065

1066

1067

1068

1069

1070

1071

1072

1073

1074

1075

1076

1077

1078

1079

1080

1081

1082

1083

1084

1086

1087

1088

1089

1090

1092

1093

1094

1095

1097

1098

1099

1100

1101

1102

1103

1104

1105

1106

1108

1109

1110

1111

1112

1113

1114

1115

1116

1117

1118

1119

1120

1121

1122

1123

1125

\section{REFERENCES}

[1] M. Salem et al., "An overview of radio resource management in relayenhanced OFDMA-based networks," IEEE Commun. Surveys Tuts., vol. 12, no. 3, pp. 422-438, Apr. 2010.

[2] L. Hanzo, Y. Akhtman, L. Wang, and M. Jiang, MIMO-OFDM for LTE, WIFI and WIMAX: Coherent Versus Non-Coherent and Cooperative Turbo-Transceivers. Hoboken, NJ, USA: Wiley, 2010, IEEE Press.

[3] C. Han et al., "Green radio: Radio techniques to enable energy-efficient wireless networks," IEEE Commun. Mag., vol. 49, no. 6, pp. 46-54, Jun. 2011.

[4] G. Caire and S. Shamai, "On the achievable throughput of a multiantenna Gaussian broadcast channel," IEEE Trans. Inf. Theory, vol. 49, no. 7, pp. 1691-1706, Jul. 2003.

[5] S. Vishwanath, N. Jindal, and A. Goldsmith, "Duality, achievable rates, and sum-rate capacity of Gaussian MIMO broadcast channels," IEEE Trans. Inf. Theory, vol. 49, no. 10, pp. 2658-2668, Oct. 2003.

[6] M. H. M. Costa, "Writing on dirty paper," IEEE Trans. Inf. Theory, vol. 29, no. 3, pp. 439-441, May 1983.

[7] T. Yoo and A. Goldsmith, "On the optimality of multiantenna broadcast scheduling using zero-forcing beamforming," IEEE J. Sel. Areas Commun., vol. 24, no. 3, pp. 528-541, Mar. 2006.

[8] N. U1 Hassan and M. Assaad, "Low complexity margin adaptive resource allocation in downlink MIMO-OFDMA system," IEEE Trans. Wireless Commun., vol. 8, no. 7, pp. 3365-3371, Jul. 2009.

[9] G. Raleigh and J. Cioffi, "Spatio-temporal coding for wireless communication,” IEEE Trans. Commun., vol. 46, no. 3, pp. 357-366, Mar. 1998.

0] K.-K. Wong, R. Murch, and K. Letaief, "A joint-channel diagonalization for multiuser MIMO antenna systems," IEEE Trans. Wireless Commun., vol. 2, no. 4, pp. 773-786, Jul. 2003.

11] W. Ho and Y.-C. Liang, "Optimal resource allocation for multiuser MIMO-OFDM systems with user rate constraints," IEEE Trans. Veh. Technol., vol. 58, no. 3, pp. 1190-1203, Mar. 2009.

12] M. Sharif and B. Hassibi, "On the capacity of MIMO broadcast channels with partial side information," IEEE Trans. Inf. Theory, vol. 51, no. 2, pp. 506-522, Feb. 2005.

13] A. Goldsmith, Wireless Communications. New York, NY, USA: Cambridge Univ. Press, 2005.

14] D. Ng, E. Lo, and R. Schober, "Energy-efficient resource allocation in multi-cell OFDMA systems with limited backhaul capacity," IEEE Trans. Wireless Commun., vol. 11, no. 10, pp. 3618-3631, Oct. 2012.

5] G. Miao, N. Himayat, and G. Li, "Energy-efficient link adaptation in frequency-selective channels," IEEE Trans. Commun., vol. 58, no. 2, pp. 545-554, Feb. 2010.

16] G. Miao, N. Himayat, G. Li, and S. Talwar, "Low-complexity energyefficient scheduling for uplink OFDMA," IEEE Trans. Commun., vol. 60, no. 1, pp. 112-120, Jan. 2012.

7] K. T. K. Cheung, S. Yang, and L. Hanzo, "Achieving maximum energy-efficiency in multi-relay OFDMA cellular networks: A fractional programming approach," IEEE Trans. Commun., vol. 61, no. 7, pp. 27462757, Jul. 2013.

18] Z. Shen, R. Chen, J. Andrews, R. Heath, and B. Evans, "Low complexity user selection algorithms for multiuser MIMO systems with block diagonalization," IEEE Trans. Signal Process., vol. 54, no. 9, pp. 3658-3663, Sep. 2006.

9] W. Yu and T. Lan, "Transmitter optimization for the multi-antenna downlink with per-antenna power constraints," IEEE Trans. Signal Process., vol. 55, no. 6, pp. 2646-2660, Jun. 2007.

0] E. Lo et al., "Adaptive resource allocation and capacity comparison of downlink multiuser MIMO-MC-CDMA and MIMO-OFDMA," IEEE Trans. Wireless Commun., vol. 6, no. 3, pp. 1083-1093, Mar. 2007.

$21]$ D. Ng, E. Lo, and R. Schober, "Dynamic resource allocation in MIMOOFDMA systems with full-duplex and hybrid relaying," IEEE Trans. Commun., vol. 60, no. 5, pp. 1291-1304, May 2012.

22] G. Brante, I. Stupia, R. D. Souza, and L. Vandendorpe, "Outage probability and energy efficiency of cooperative MIMO with antenna selection," IEEE Trans. Wireless Commun., vol. 12, no. 11, pp. 5896-5907, Nov. 2013.
[23] A. Zappone, P. Cao, and E. Jorswieck, "Energy efficiency optimization 1126 in relay-assisted MIMO systems with perfect and statistical CSI," IEEE 1127 Trans. Signal Process., vol. 62, no. 2, pp. 443-457, Jan. 2014.

[24] M. Avriel, W. E. Diewert, S. Schaible, and I. Zang, Generalized Concav- 1129 ity. New York, NY, USA: Plenum, 1988.

1130

[25] R. Devarajan, S. Jha, U. Phuyal, and V. Bhargava, "Energy-aware re- 1131 source allocation for cooperative cellular network using multi-objective 1132 optimization approach," IEEE Trans. Wireless Commun., vol. 11, no. 5, 1133 pp. 1797-1807, May 2012.

1134

[26] W. Dinkelbach, "On nonlinear fractional programming," Manag. Sci., 1135 vol. 13, no. 7, pp. 492-498, Mar. 1967.

1136

[27] D. Ng, E. Lo, and R. Schober, "Energy-efficient resource allocation for 1137 secure OFDMA systems," IEEE Trans. Veh. Technol., vol. 61, no. 6, 1138 pp. 2572-2585, Jul. 2012

1139

[28] C. Isheden, Z. Chong, E. Jorswieck, and G. Fettweis, "Framework 1140 for link-level energy efficiency optimization with informed transmitter," 1141 IEEE Trans. Wireless Commun., vol. 11, no. 8, pp. 2946-2957, Aug. 2012. 1142

[29] J. Laneman, D. Tse, and G. Wornell, "Cooperative diversity in wireless 1143 networks: Efficient protocols and outage behavior," IEEE Trans. Inf. The- 1144 ory, vol. 50, no. 12, pp. 3062-3080, Dec. 2004.

[30] "Further advancements for e-utra, physical layer aspects (Release 9)," 1146 Sophia-Antipolis Cedex, France, TR 36.814 V9.0.0, Mar. 2010.

1147

[31] S.-J. Kim, A. Magnani, A. Mutapcic, S. Boyd, and Z.-Q. Luo, "Robust 1148 beamforming via worst-case SINR maximization," IEEE Trans. Signal 1149 Process., vol. 56, no. 4, pp. 1539-1547, Apr. 2008.

1150

[32] S. Boyd and L. Vandenberghe, Convex Optimization. New York, NY, 1151 USA: Cambridge Univ. Press, 2004.

1152

[33] L. Hanzo, O. Alamri, M. El-Hajjar, and N. Wu, Near-Capacity Multi- 1153 Functional MIMO Systems: Sphere-Packing, Iterative Detection and Co- 1154 operation. Hoboken, NJ, USA: Wiley, 2009, IEEE Press.

1155

[34] A. Yeredor, "Non-orthogonal joint diagonalization in the least-squares 1156 sense with application in blind source separation," IEEE Trans. Signal 1157 Process., vol. 50, no. 7, pp. 1545-1553, Jul. 2002.

1158

[35] P. Comon and C. Jutten, Handbook of Blind Source Separation: Inde- 1159 pendent Component Analysis and Applications. New York, NY, USA: 1160 Academic, 2010.

1161

[36] G. Auer et al., "D2.3: Energy Efficiency Analysis of the Reference Sys- 1162 tems, Areas of Improvements and Target Breakdown," Tech. Rep. INFSO- 1163 ICT-247733, Nov. 2010. [Online]. Available: https://bscw.ict-earth.eu/ 1164 AQ1 pub/bscw.cgi/d71252/EARTH_WP2_D2.3_v2.pdf 1165

[37] D. P. Bertsekas, Nonlinear Programming. Belmont, MA, USA: Athena 1166 Scientific, 1999.

1167

[38] W. Yu and R. Lui, "Dual methods for nonconvex spectrum optimization 1168 of multicarrier systems," IEEE Trans. Commun., vol. 54, no. 7, pp. 1310- 1169 1322, Jul. 2006.

1170

[39] K. Seong, M. Mohseni, and J. Cioffi, "Optimal resource allocation for 1171 OFDMA downlink systems," in Proc. IEEE ISIT, Seattle, Washington, 1172 USA, Jul. 2006, pp. 1394-1398.

1173

[40] D. Ng and R. Schober, "Cross-layer scheduling for OFDMA amplify- 1174 and-forward relay networks," IEEE Trans. Veh. Technol., vol. 59, no. 3, 1175 pp. 1443-1458, Mar. 2010.

1176

[41] D. Palomar and M. Chiang, "A tutorial on decomposition methods for 1177 network utility maximization," IEEE J. Sel. Areas Commun., vol. 24, 1178 no. 8, pp. 1439-1451, Aug. 2006

1179

[42] O. Arnold, F. Richter, G. Fettweis, and O. Blume, "Power consumption 1180 modeling of different base station types in heterogeneous cellular net- 1181 works," in Proc. Future Netw. Mobile Summit, Florence, Italy, Jun. 2010, 1182 pp. 1-8.

1183

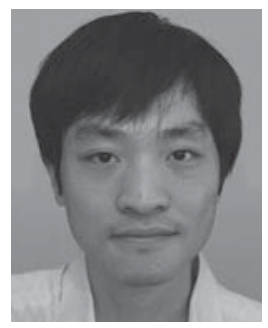

Kent Tsz Kan Cheung (S'09) received the B.Eng. 1184 degree (first-class honors) in electronic engineering 1185 from the Univeristy of Southampton, Southampton, 1186 U.K., in 2009. Since then he has been pursuing the 1187 Ph.D. degree in wireless communications at the same 1188 institution. He was a recipient of the EPSRC Indus- 1189 trial CASE award in 2009, and was involved with the 1190 Core 5 Green Radio project of the Virtual Centre of 1191 Excellence in Mobile and Personal Communications 1192 (Mobile VCE).

1193

His research interests include energy-efficiency, 1194 multi-carrier MIMO communications, cooperative communications, resource 1195 allocation and optimization. 


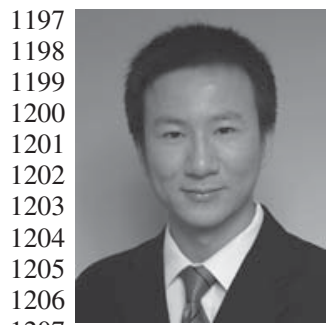

1207

1208 Southampton, U.K.

1209 From November 2008 to February 2009, he was an Intern Research Fellow 1210 with the Communications Technology Lab (CTL), Intel Labs, Beijing, China, 1211 where he focused on Channel Quality Indicator Channel (CQICH) design for 1212 mobile WiMAX (802.16 m) standard. His research interests include Multiple1213 Input-Multiple Output (MIMO) signal processing, multicell joint/distributed 1214 signal processing, cooperative communications, green radio and cross-layer 1215 interference management. He has published more than 30 research papers on 1216 IEEE journals and conferences.

1217 Shaoshi received a number of awards, such as the PMC-Sierra Telecom1218 munications Technology Scholarship, the Electronics and Computer Science 1219 Research Studentship and the China Scholarship Council Scholarship. He 1220 is a member of IEEE/IET, and a junior member of Isaac Newton Institute 1221 for Mathematical Sciences, Cambridge, U.K. He is also a TPC member of 1222 several major IEEE journals/conferences, including PIMRC 2012, ICC 2013, 1223 ICCVE 2013, ICCVE 2014, ICC 2015 and IEEE JOURNAL ON SELECTED 1224 AREAS IN COMMUNiCATIONS-SPECIAL ISSUE ON RECENT ADVANCES IN 1225 Heterogeneous Cellular NeTwORKS.

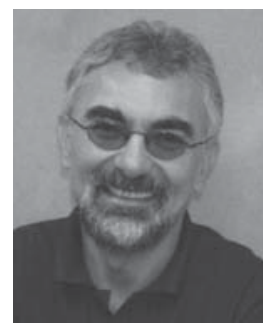

Lajos Hanzo (F'XX) (http://www.cspc.ecs.soton.ac. 1226 AQ2 uk) FREng, FIEEE, FIET, Fellow of EURASIP, 1227 DSc received the degree in electronics in 1976 and 1228 the doctorate in 1983. In 2009 he was awarded the 1229 honorary doctorate Doctor Honoris Causa by the 1230 Technical University of Budapest.

During his 37-year career in telecommunications 1232 he has held various research and academic posts in 1233 Hungary, Germany and the UK. Since 1986 he has 1234 been with the School of Electronics and Computer 1235 Science, University of Southampton, UK, where he 1236 holds the chair in telecommunications. He has successfully supervised $80+1237$ $\mathrm{PhD}$ students, co-authored 20 John Wiley/IEEE Press books on mobile radio 1238 communications totalling in excess of 10000 pages, published $1400+$ research 1239 entries at IEEE Xplore, acted both as TPC and General Chair of IEEE 1240 conferences, presented keynote lectures and has been awarded a number of 1241 distinctions. Currently he is directing a 100-strong academic research team, 1242 working on a range of research projects in the field of wireless multimedia 1243 communications sponsored by industry, the Engineering and Physical Sciences 1244 Research Council (EPSRC) UK, the European Research Council's Advanced 1245 Fellow Grant and the Royal Society's Wolfson Research Merit Award.

1246

$\mathrm{He}$ is an enthusiastic supporter of industrial and academic liaison and he 1247 offers a range of industrial courses. He is also a Governor of the IEEE VTS. 1248 During 2008-2012 he was the Editor-in-Chief of the IEEE Press and a Chaired 1249 Professor also at Tsinghua University, Beijing. His research is funded by 1250 the European Research Council's Senior Research Fellow Grant. For further 1251 information on research in progress and associated publications please refer to 1252 http://www.cspc.ecs.soton.ac.uk Lajos has 20000+ citations. 


\section{AUTHOR QUERIES}

\section{AUTHOR PLEASE ANSWER ALL QUERIES}

AQ1 = Please provide organizational address [36].

AQ2 = Please provide membership history of author Lajos Hanzo.

\section{END OF ALL QUERIES}

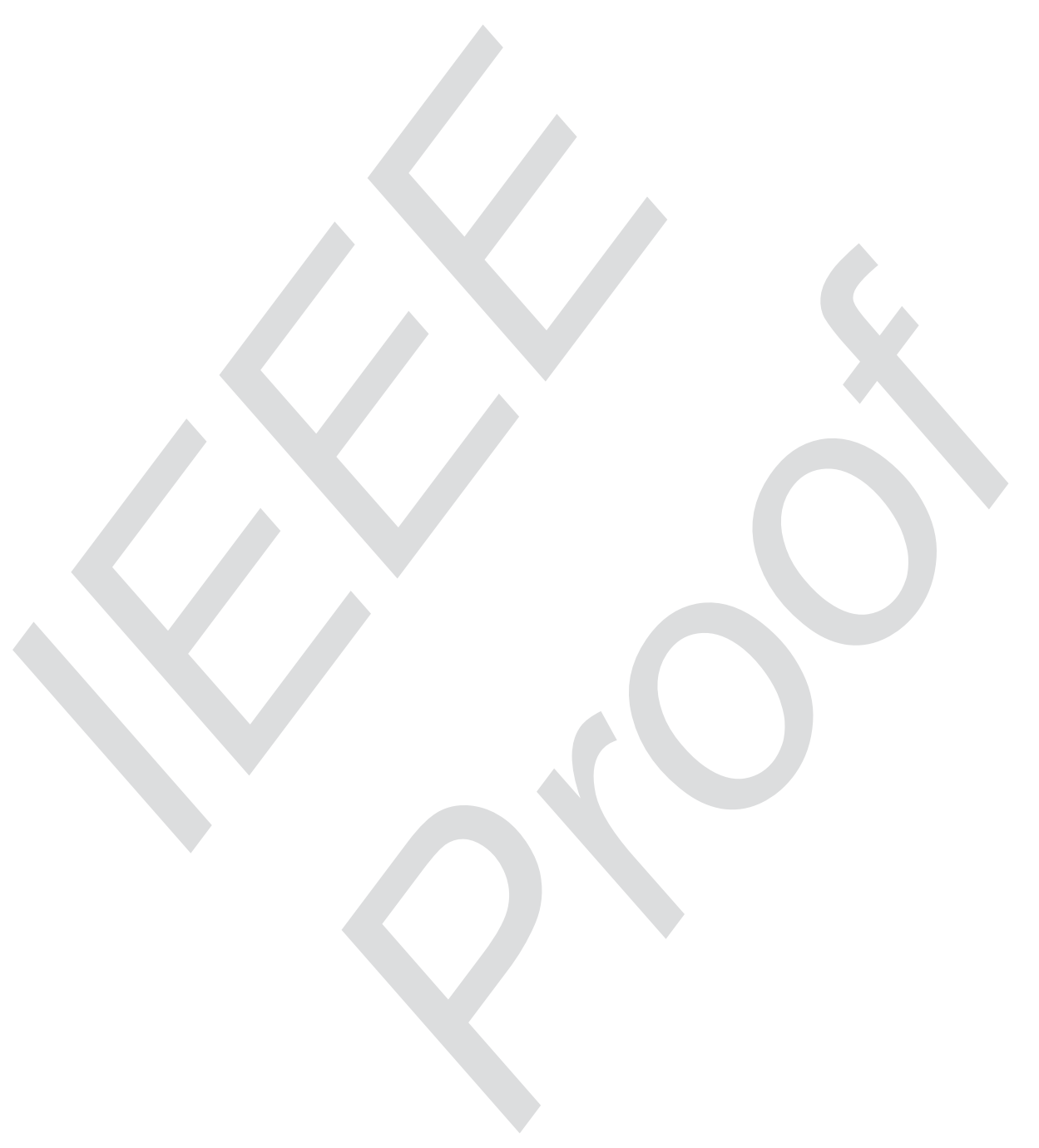

